Supporting Information for

\title{
Sequential and Diverse Synthesis of BN-Heterocycles and Investigation of Their Photoreactivity
}

\author{
Deng-Tao Yang, a, b, c, * Jie Zheng, ${ }^{\mathrm{c}}$ Jin-Bao Peng, ${ }^{\mathrm{c}}$ Xiang Wang ${ }^{\mathrm{c}}$ and Suning Wang ${ }^{\mathrm{c}}$
}

anstitute of Advanced Synthesis, Northwestern Polytechnical University, Xi'an, 710072, China.

Corresponding author: D.-T. Yang, email: dtyang@nwpu.edu.cn

${ }^{\text {b} Y a n g t z e ~ R i v e r ~ D e l t a ~ R e s e a r c h ~ I n s t i t u t e, ~ N o r t h w e s t e r n ~ P o l y t e c h n i c a l ~ U n i v e r s i t y, ~ T a i c a n g ~ J i a n g s u, ~} 215400$, China.

'Department of Chemistry, Queen's University, Kingston, Ontario K7L 3N6, Canada

Contents

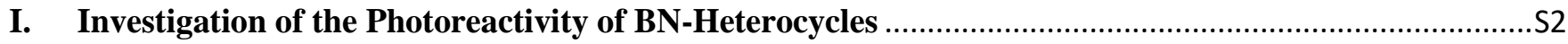

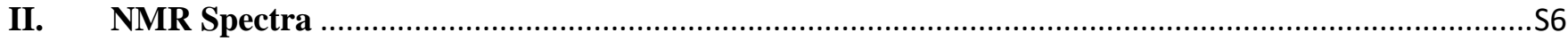

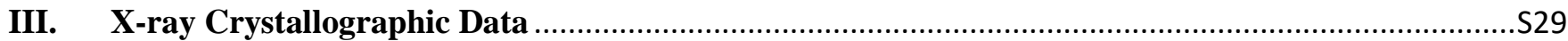




\section{Investigation of the Photoreactivity of $\mathrm{BN}$-Heterocycles}

3.1 Photoreactivity of BN-Heterocycle DBN-1 and Formation of Azaborine DBN-1a

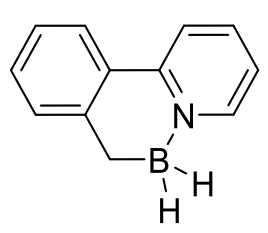

DBN-1

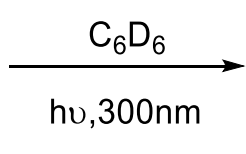

hu, $300 \mathrm{~nm}$

(1)

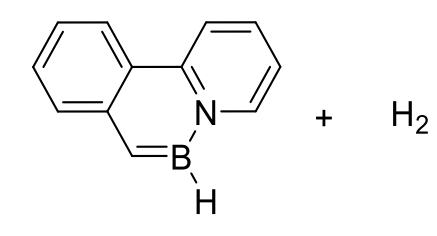

DBN-1a

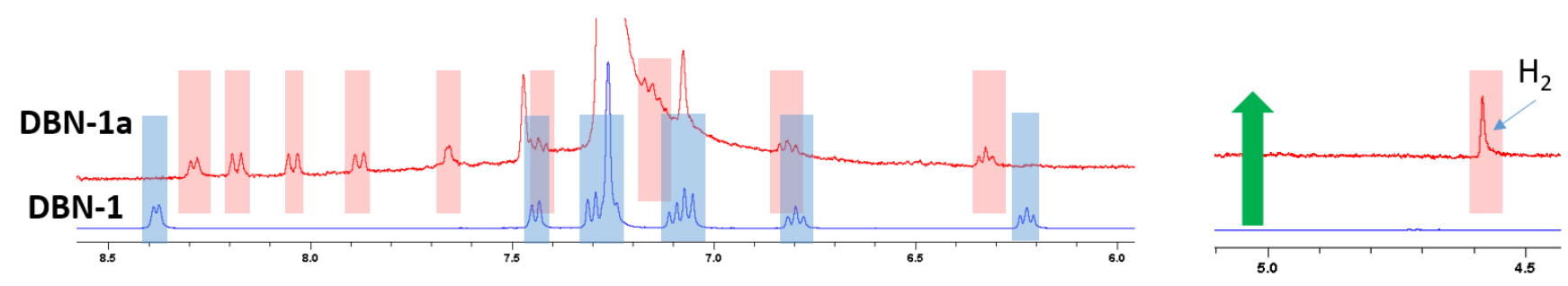

DBN-1a

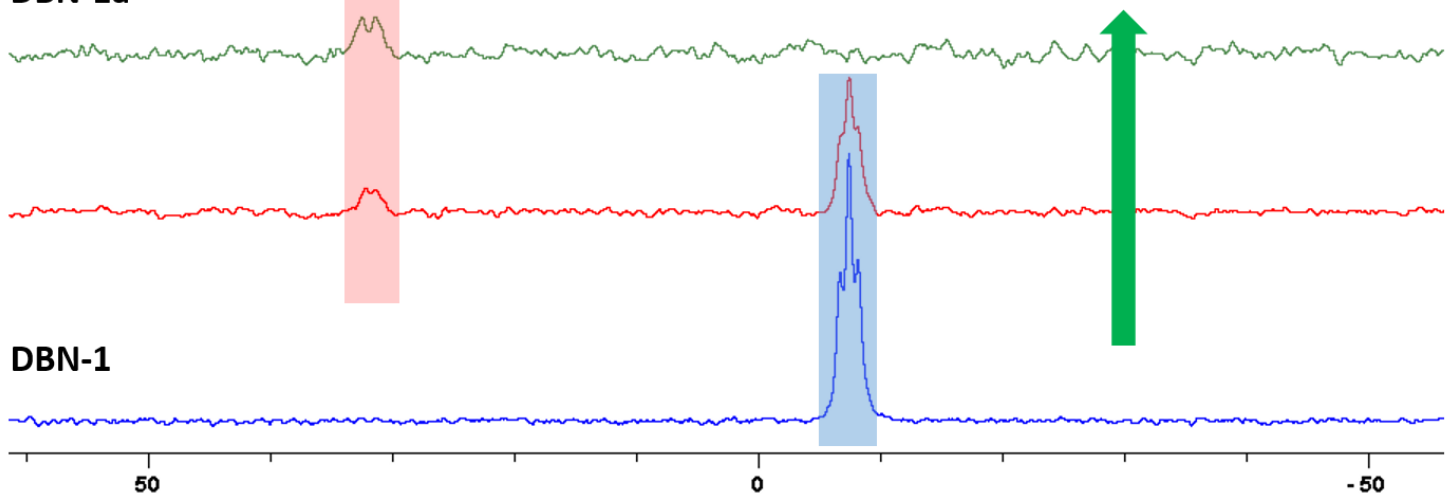


3.2 Photoreactivity of BN-Heterocycle DBN-7 and Formation of Azaborine DBN-7a
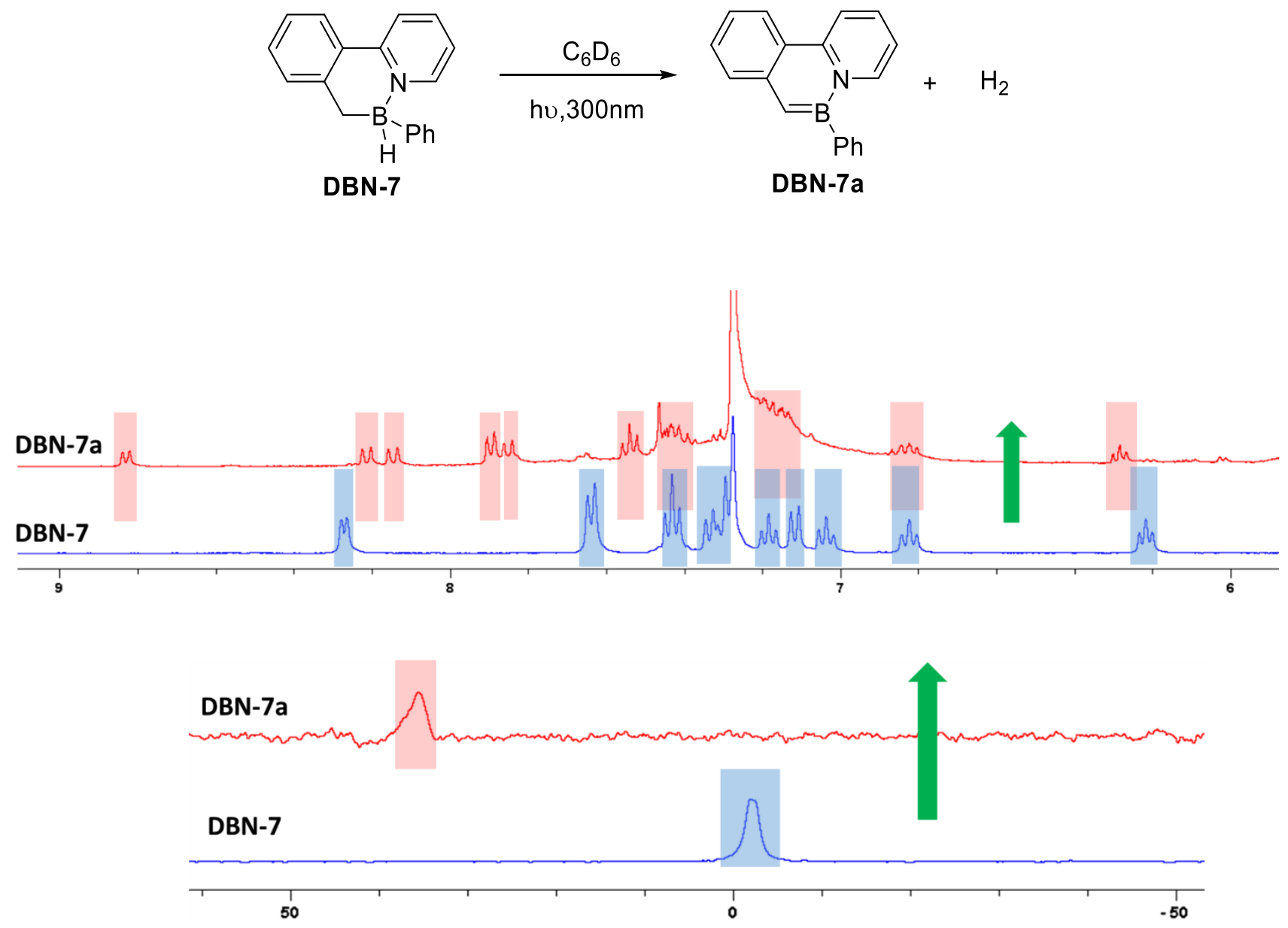
3.3 Photoreactivity of BN-Heterocycle DBN-10 and Formation of Azaborine DBN-10a
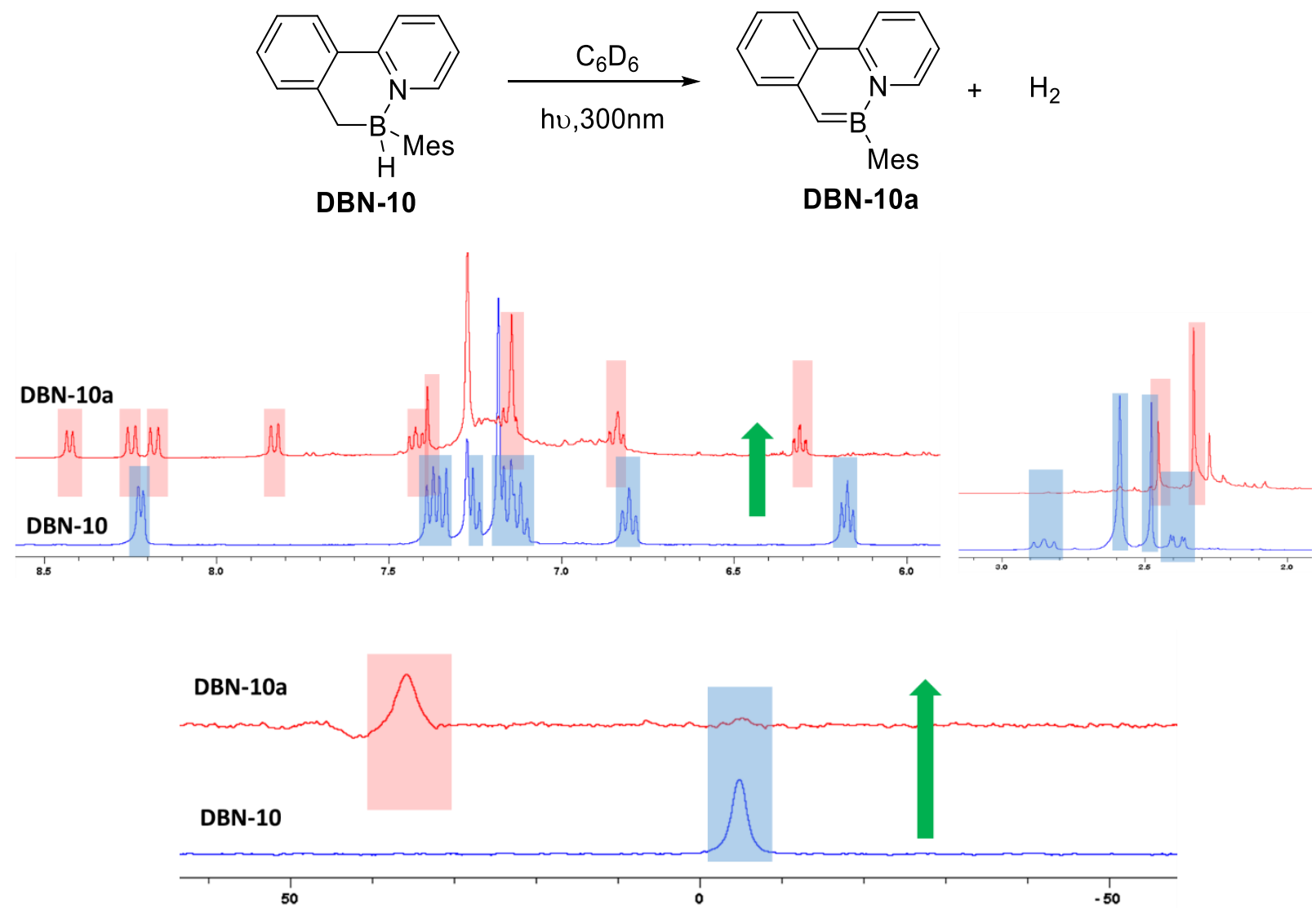

The data of DBN-10a is the same to the one reported by our group previously. 
3.4 Photoreactivity of BN-Heterocycle DBN-12 and Formation of Azaborine DBN-12a
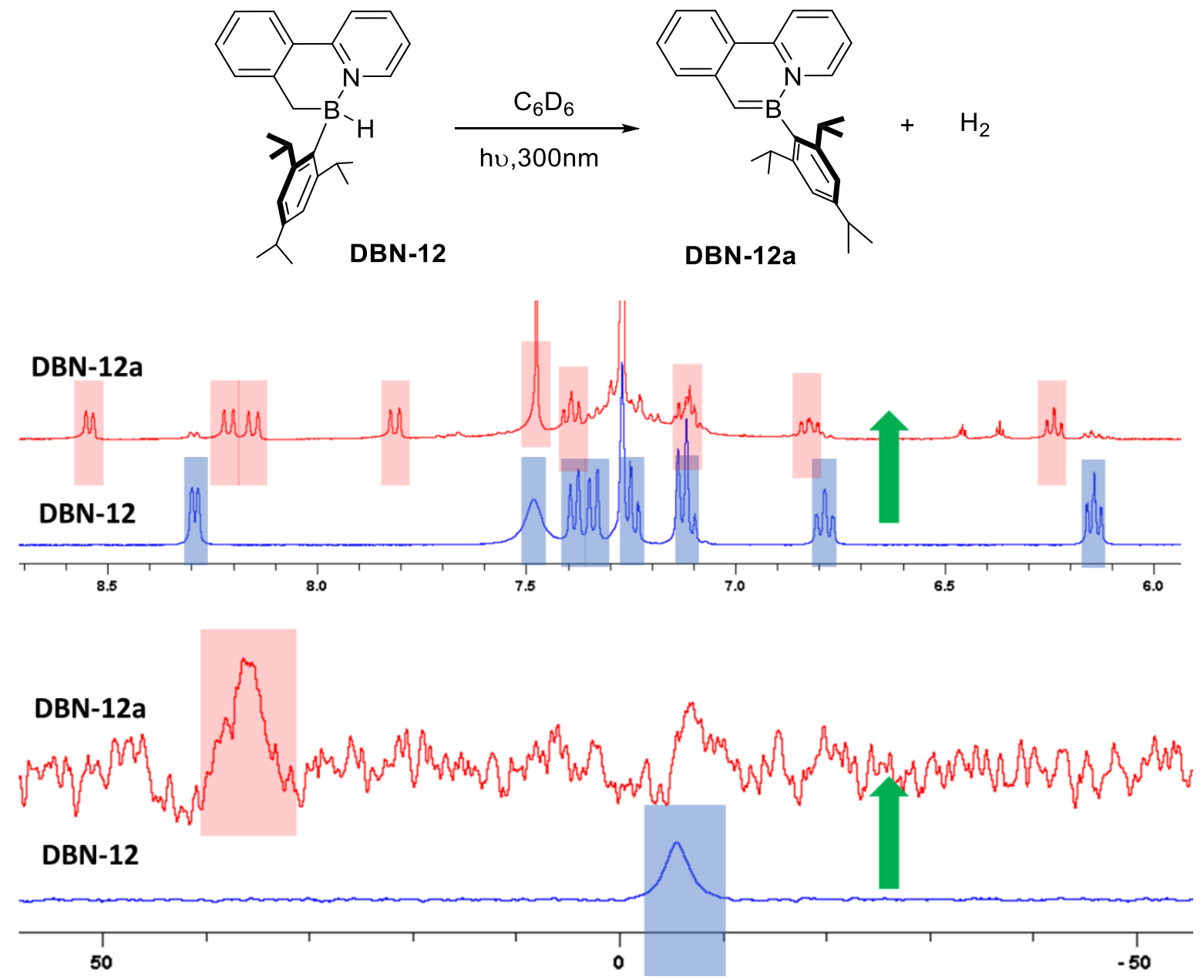

The data of DBN-12a is the same to the one reported by our group previously. 


\section{NMR Spectra}

$2.1{ }^{1} \mathrm{H}(400 \mathrm{MHz}),{ }^{13} \mathrm{C}(101 \mathrm{MHz})$ and ${ }^{11} \mathrm{~B}(128 \mathrm{MHz})$ NMR of BN-heterocycle DBN-1 in $\mathrm{CDCl}_{3}$

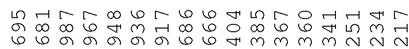

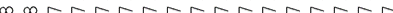

$\longrightarrow$
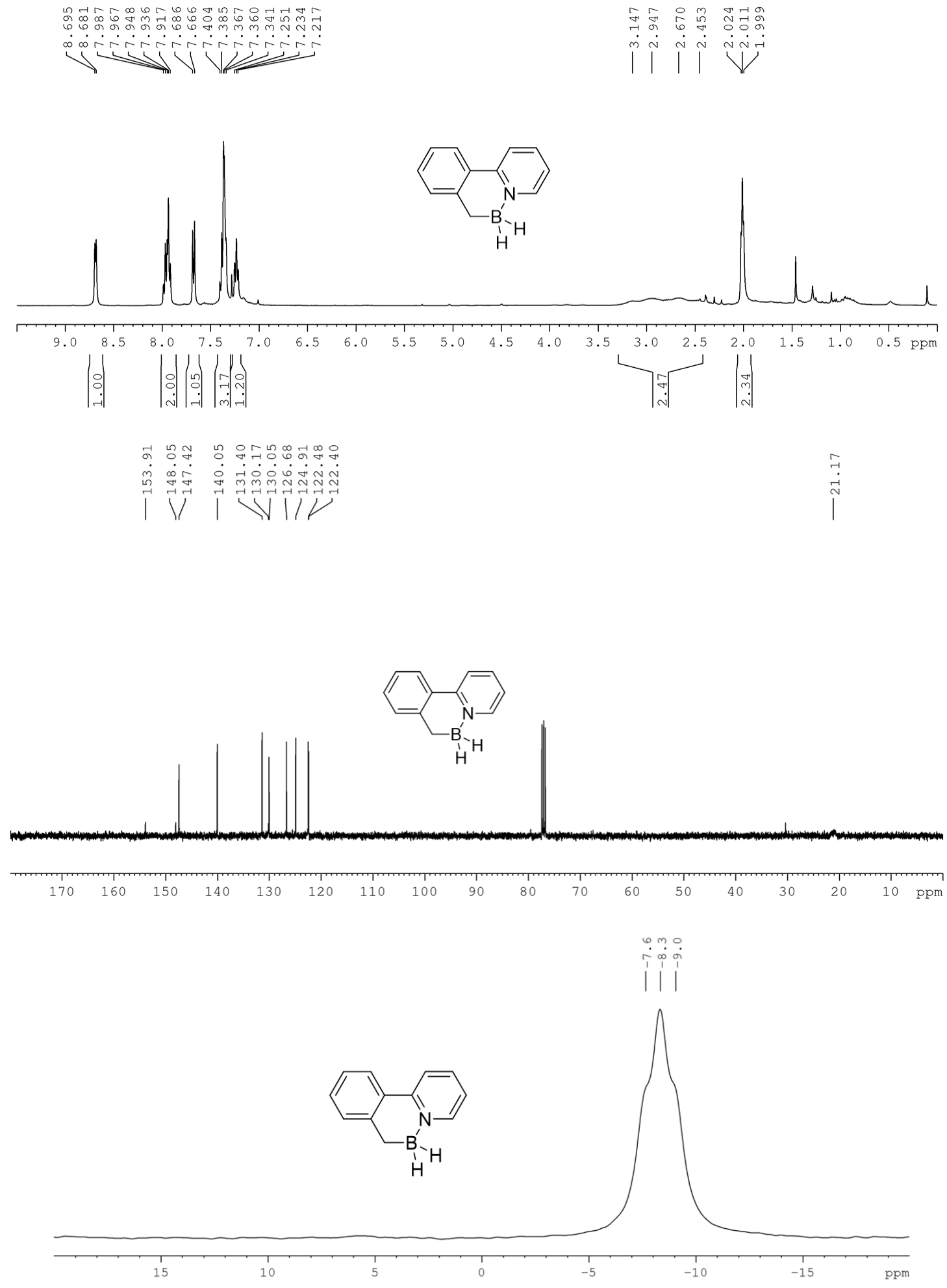


\section{$2.2{ }^{1} \mathrm{H}(400 \mathrm{MHz}),{ }^{13} \mathrm{C}(101 \mathrm{MHz})$ and ${ }^{11} \mathrm{~B}(128 \mathrm{MHz})$ NMR of BN-heterocycle DBN-2 in CDCl 3}

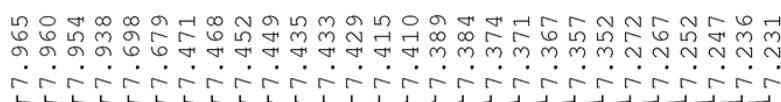

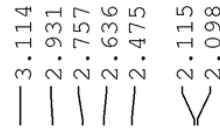

ฟึ
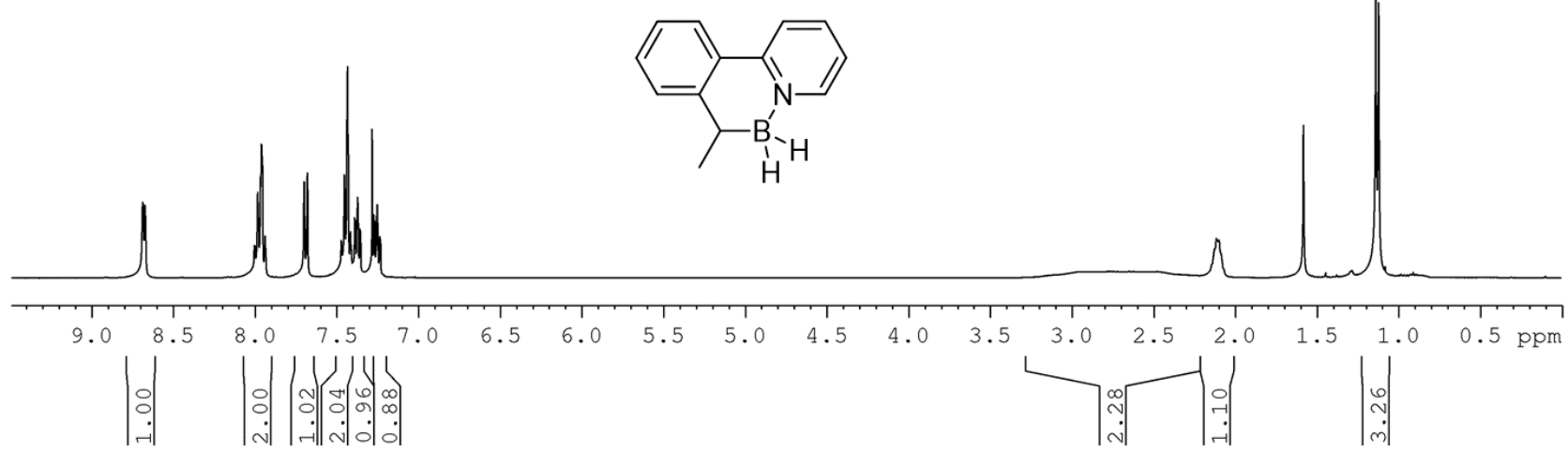

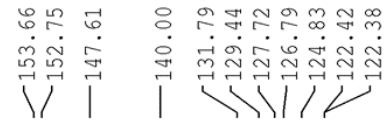
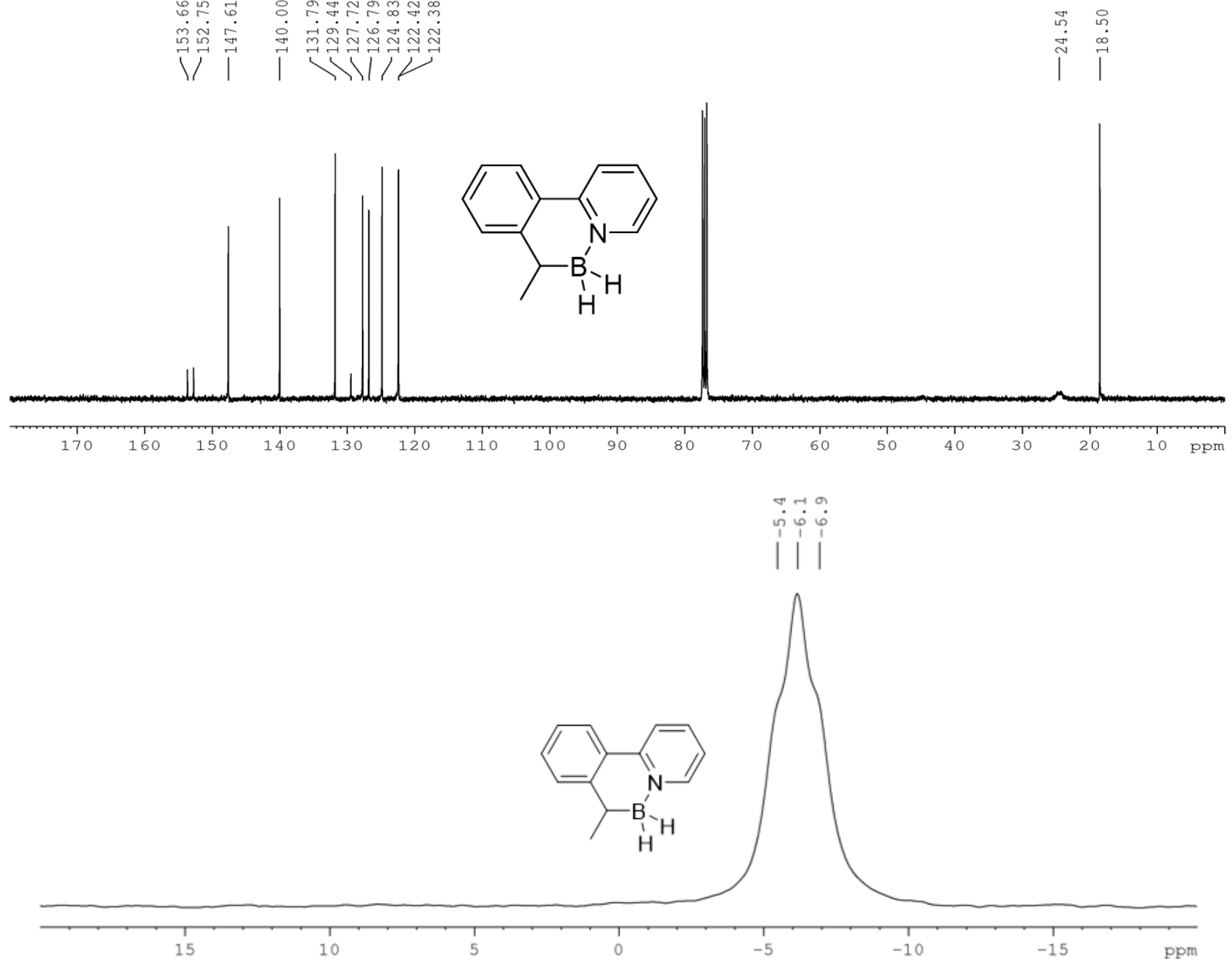
$2.3{ }^{1} \mathrm{H}(400 \mathrm{MHz}),{ }^{13} \mathrm{C}(101 \mathrm{MHz})$ and ${ }^{11} \mathrm{~B}(160 \mathrm{MHz}) \mathrm{NMR}$ of BN-heterocycle DBN-3 in $\mathrm{CDCl}_{3}\left({ }^{11} \mathrm{~B}\right.$ $\mathrm{NMR}$ in $\mathrm{C}_{6} \mathrm{D}_{6}$ )
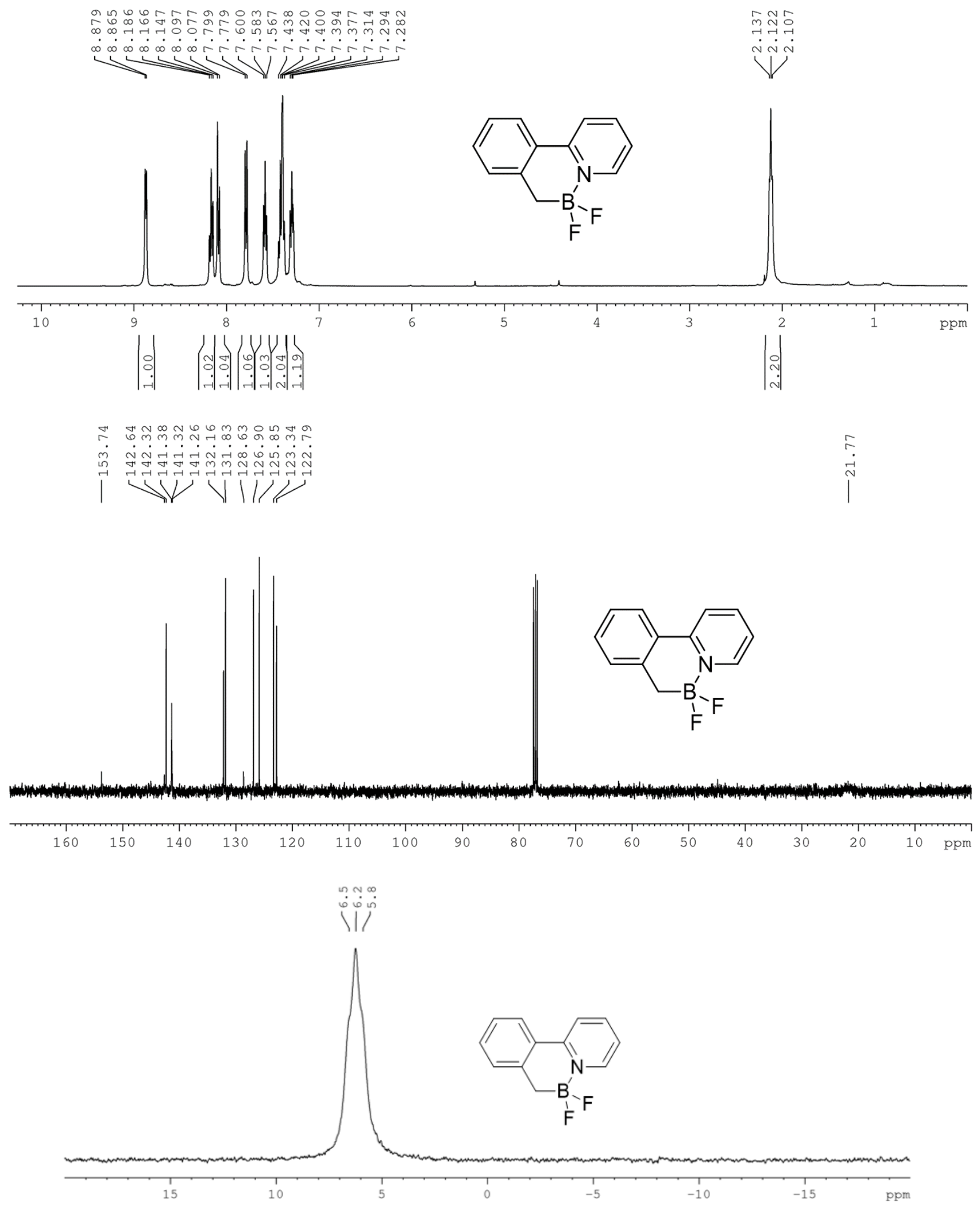


\section{$2.4{ }^{1} \mathrm{H}(400 \mathrm{MHz}),{ }^{13} \mathrm{C}(101 \mathrm{MHz})$ and ${ }^{11} \mathrm{~B}(128 \mathrm{MHz})$ NMR of BN-heterocycle DBN-4 in $\mathrm{CDCl}_{3}$}
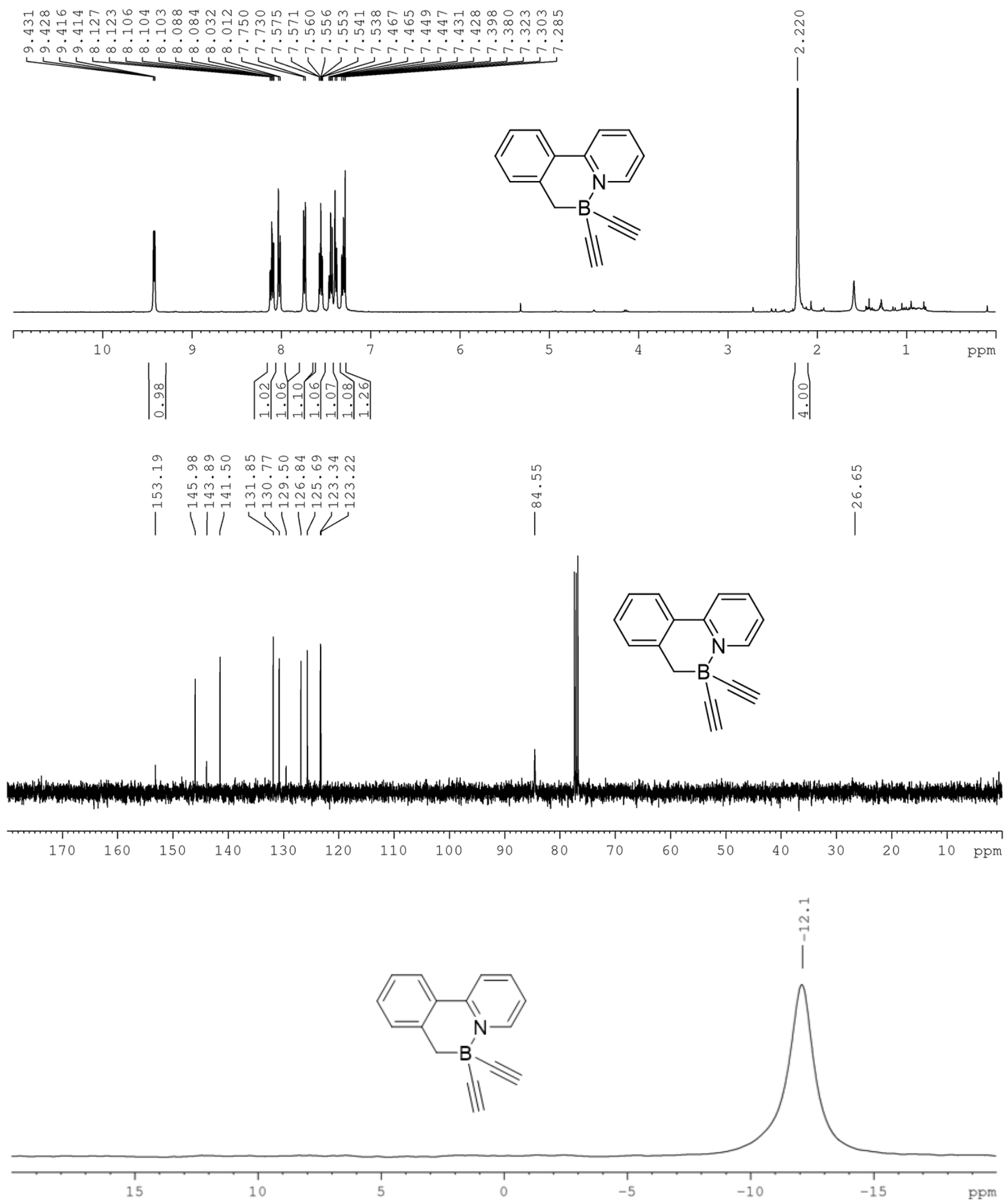
$2.5{ }^{1} \mathrm{H}(400 \mathrm{MHz}){ }^{13} \mathrm{C}(101 \mathrm{MHz})$ and ${ }^{11} \mathrm{~B}(128 \mathrm{MHz})$ NMR of BN-heterocycle DBN-4-'Bu in CDCl 3

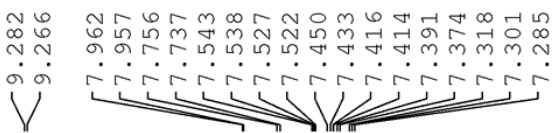
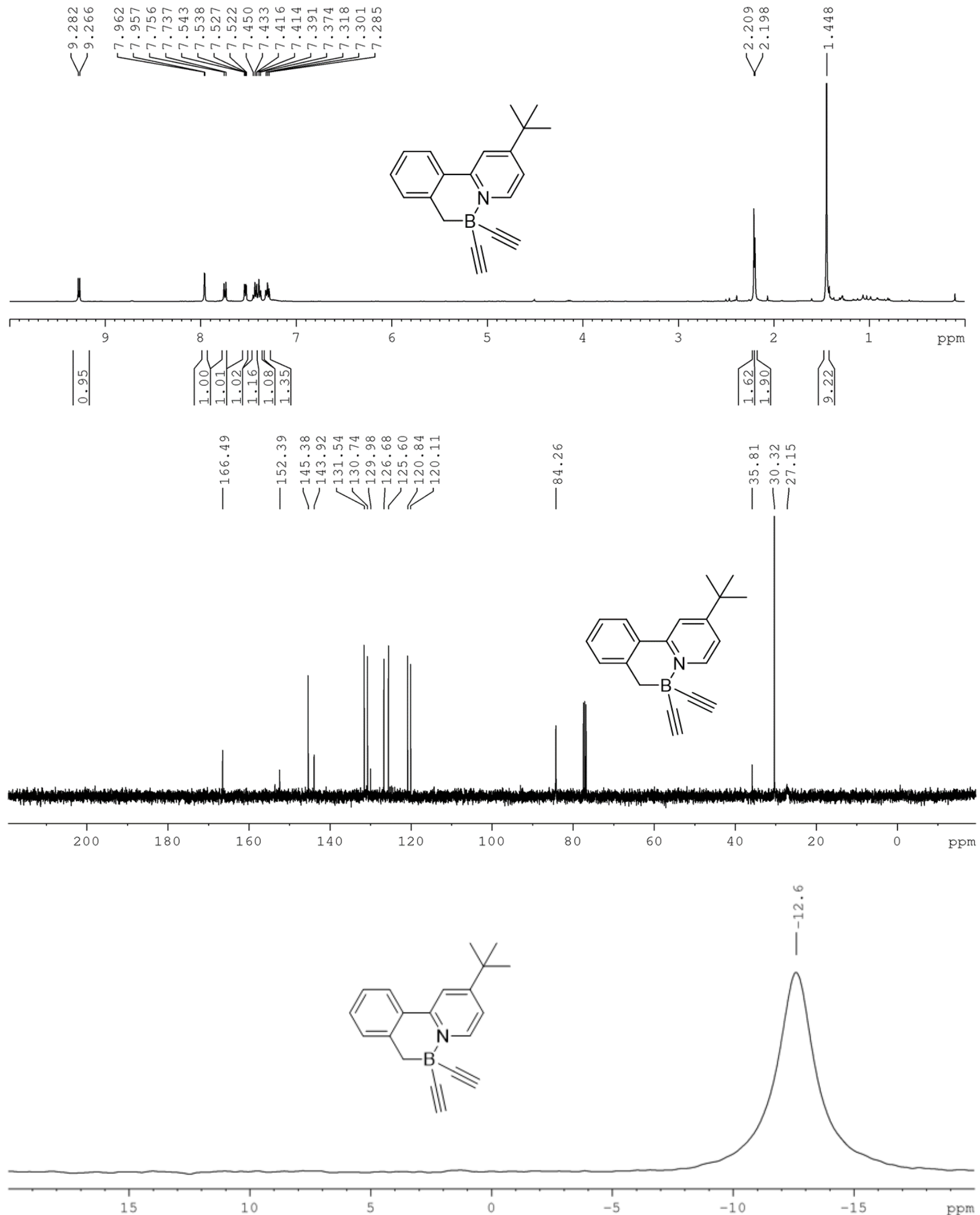


\section{$2.6{ }^{1} \mathrm{H}(400 \mathrm{MHz}),{ }^{13} \mathrm{C}(101 \mathrm{MHz})$ and ${ }^{11} \mathrm{~B}(128 \mathrm{MHz})$ NMR of BN-heterocycle DBN-5 in $\mathrm{C}_{6} \mathrm{D}_{6}$}

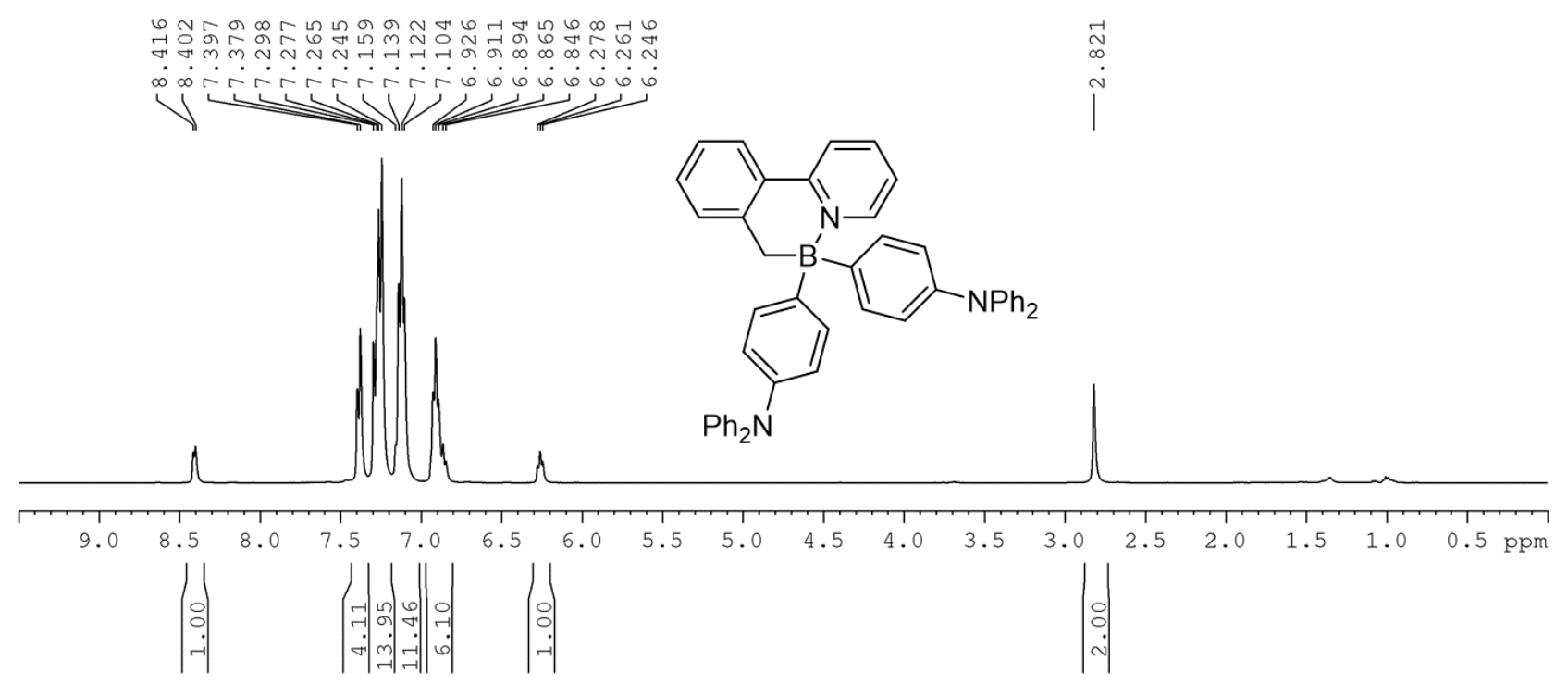

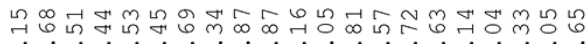

แُ

$+$
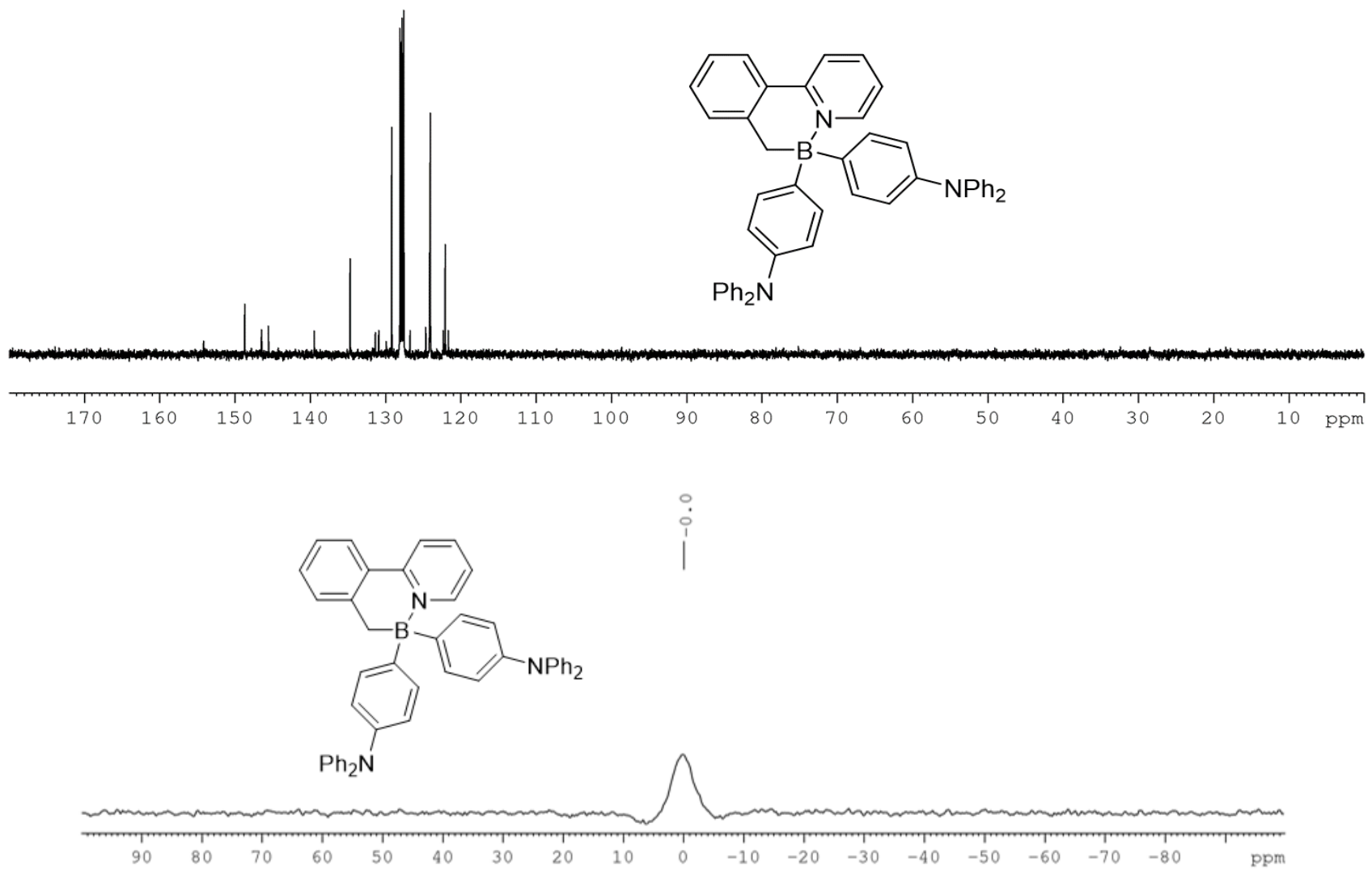
$2.7{ }^{1} \mathrm{H}(400 \mathrm{MHz}),{ }^{13} \mathrm{C}(101 \mathrm{MHz})$ and ${ }^{11} \mathrm{~B}(128 \mathrm{MHz})$ NMR of BN-heterocycle DBN-6 in $\mathrm{CDCl}_{3}$

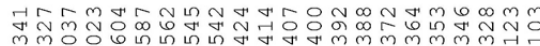

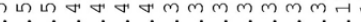

on
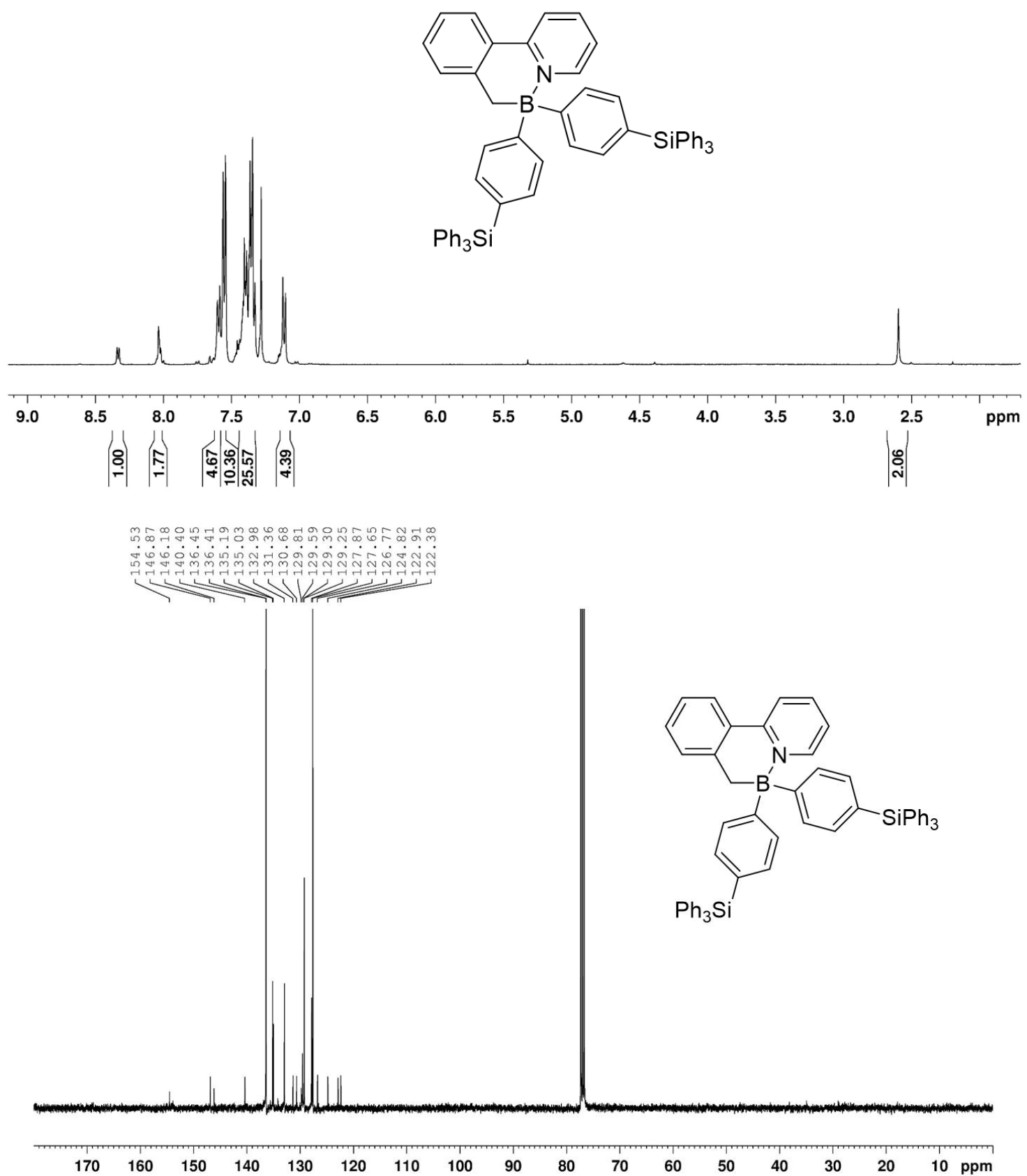
$2.8{ }^{1} \mathrm{H}\left(400 \mathrm{MHz}, \mathrm{C}_{6} \mathrm{D}_{6}\right),{ }^{13} \mathrm{C}\left(101 \mathrm{MHz}, \mathrm{CDCl}_{3}\right)$ and ${ }^{11} \mathrm{~B}\left(128 \mathrm{MHz}, \mathrm{C}_{6} \mathrm{D}_{6}\right)$ NMR of BN-heterocycle DBN-7
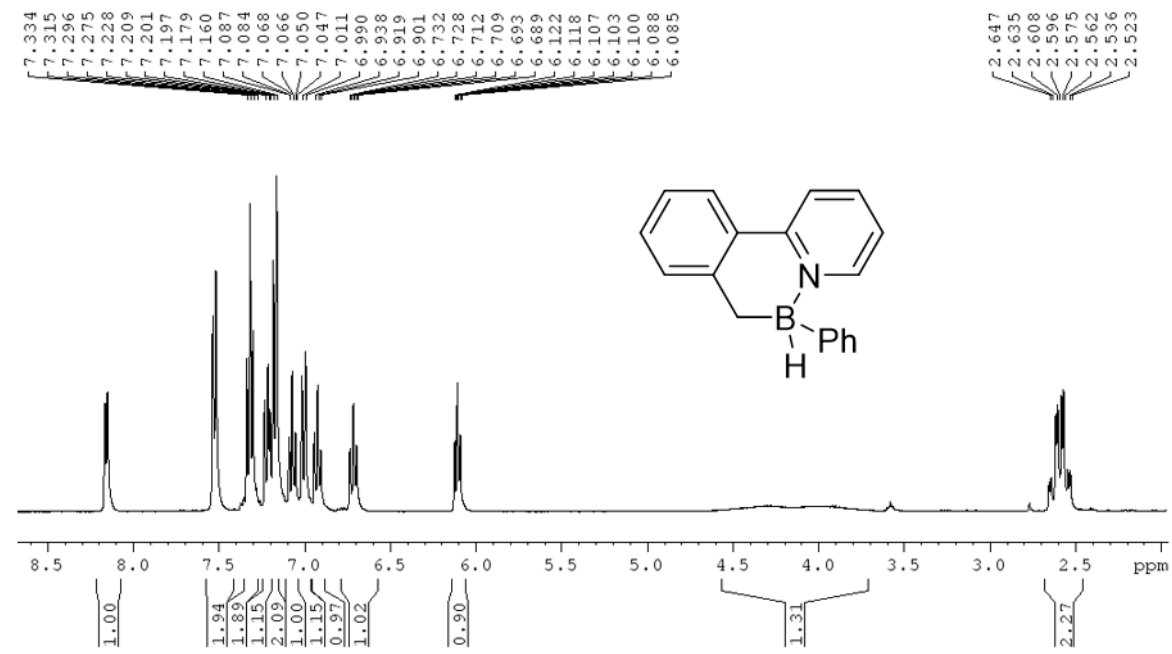

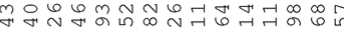

东本m

II
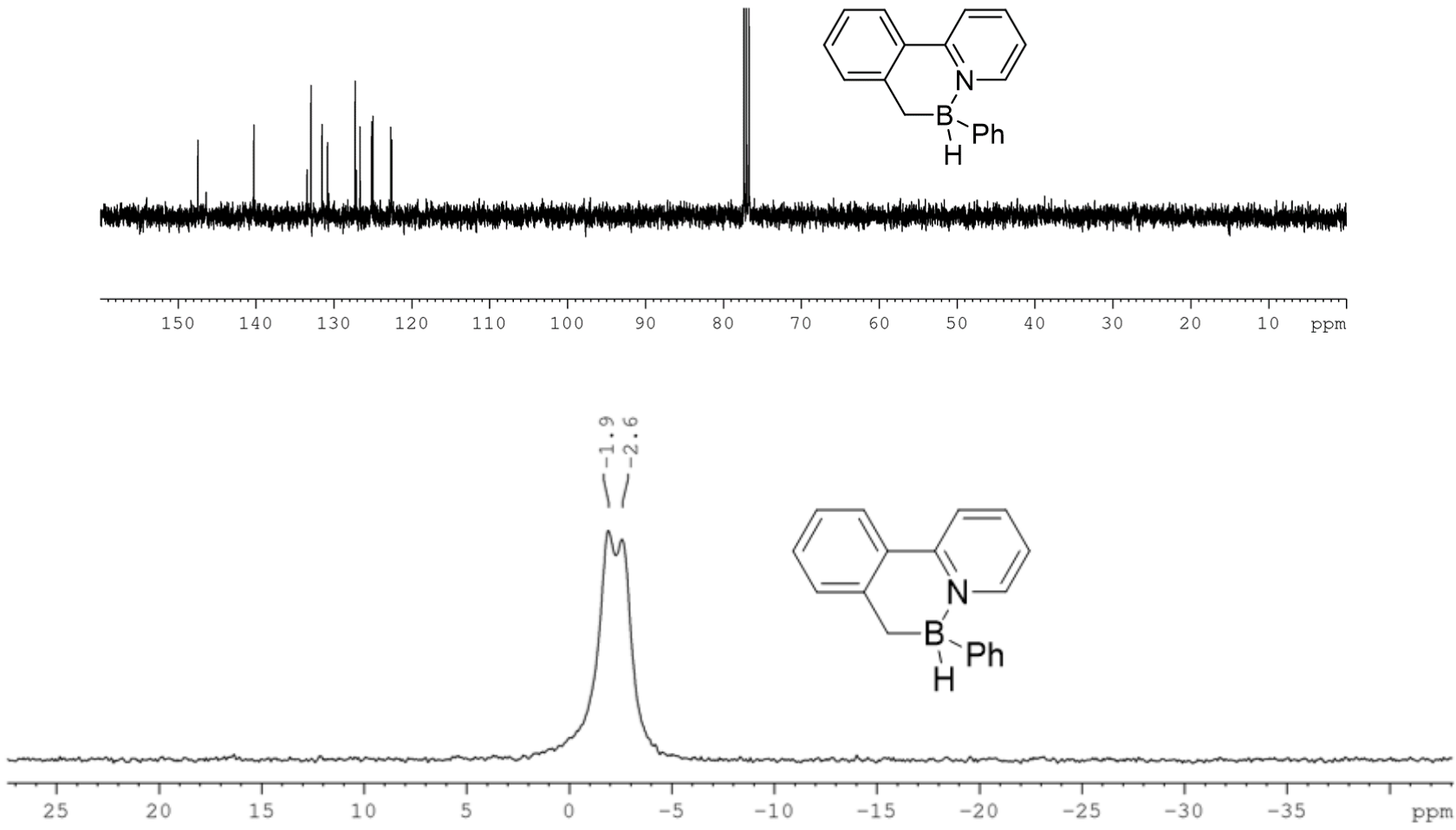


\section{$2.9{ }^{1} \mathrm{H}(400 \mathrm{MHz}),{ }^{13} \mathrm{C}(101 \mathrm{MHz})$ and ${ }^{11} \mathrm{~B}(128 \mathrm{MHz}) \mathrm{NMR}$ of BN-heterocycle DBN-8 in $\mathrm{CDCl}_{3}$}
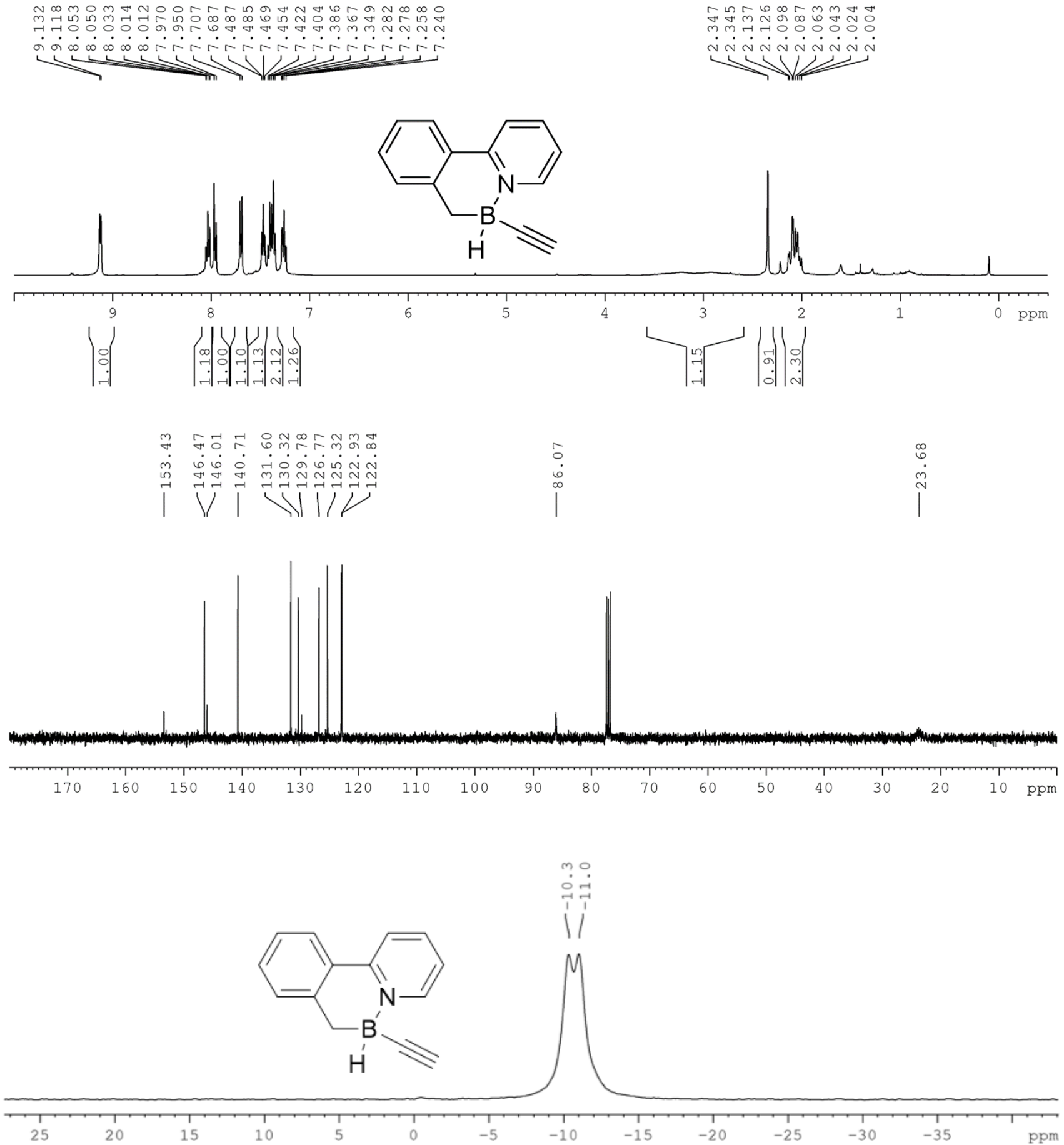
$2.10{ }^{1} \mathrm{H}(400 \mathrm{MHz}),{ }^{13} \mathrm{C}(101 \mathrm{MHz})$ and ${ }^{11} \mathrm{~B}(128 \mathrm{MHz})$ NMR of BN-heterocycle DBN-9 in $\mathrm{CDCl}_{3}$
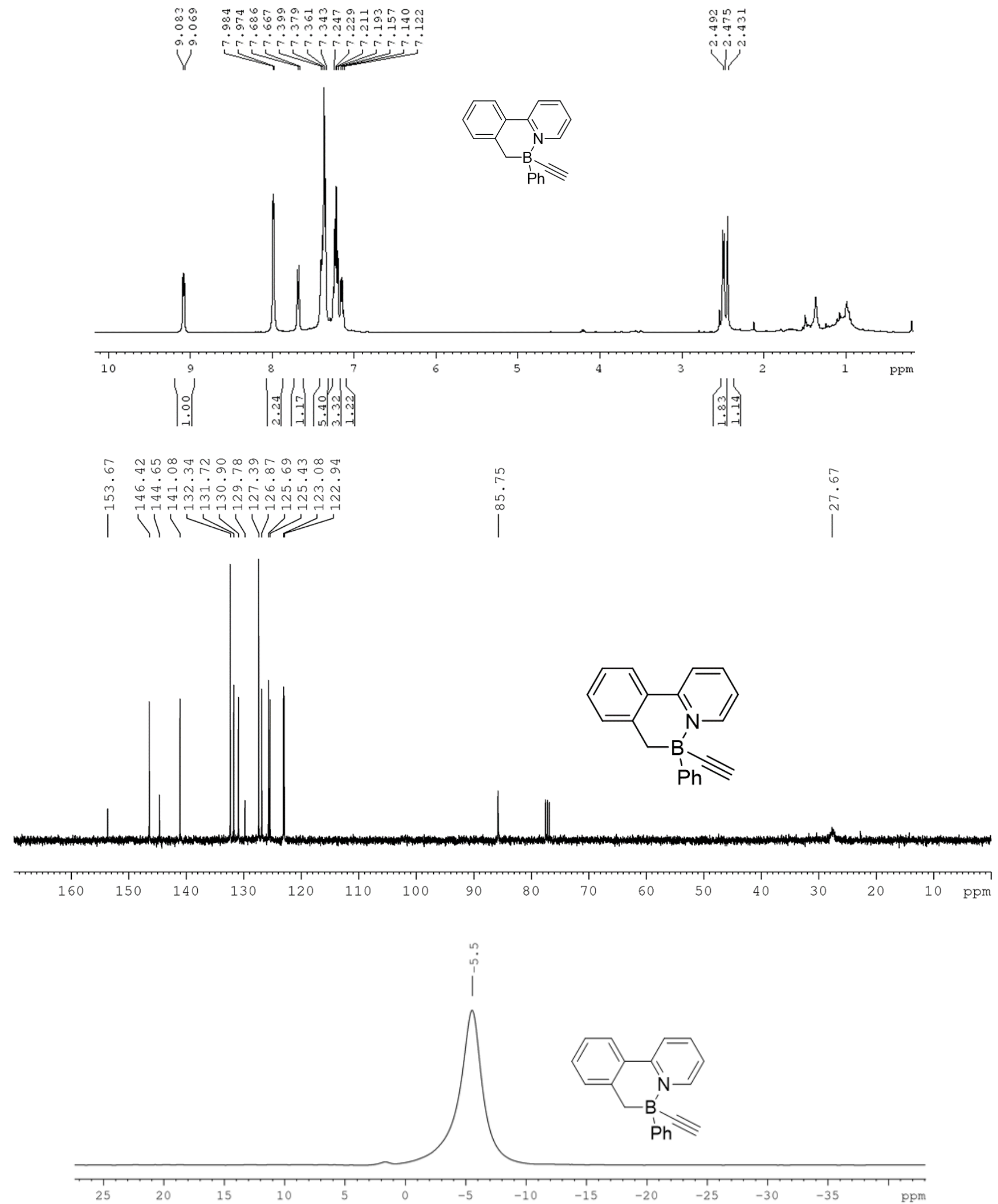
$2.11{ }^{1} \mathrm{H}(499 \mathrm{MHz}),{ }^{13} \mathrm{C}(126 \mathrm{MHz})$ and ${ }^{11} \mathrm{~B}(160 \mathrm{MHz}) \mathrm{NMR}$ of $\mathrm{BN}$-heterocycle $\mathrm{DBN}-10$ in $\mathrm{CDCl}_{3}$

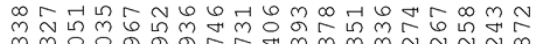

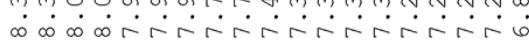

$\longrightarrow$ W W

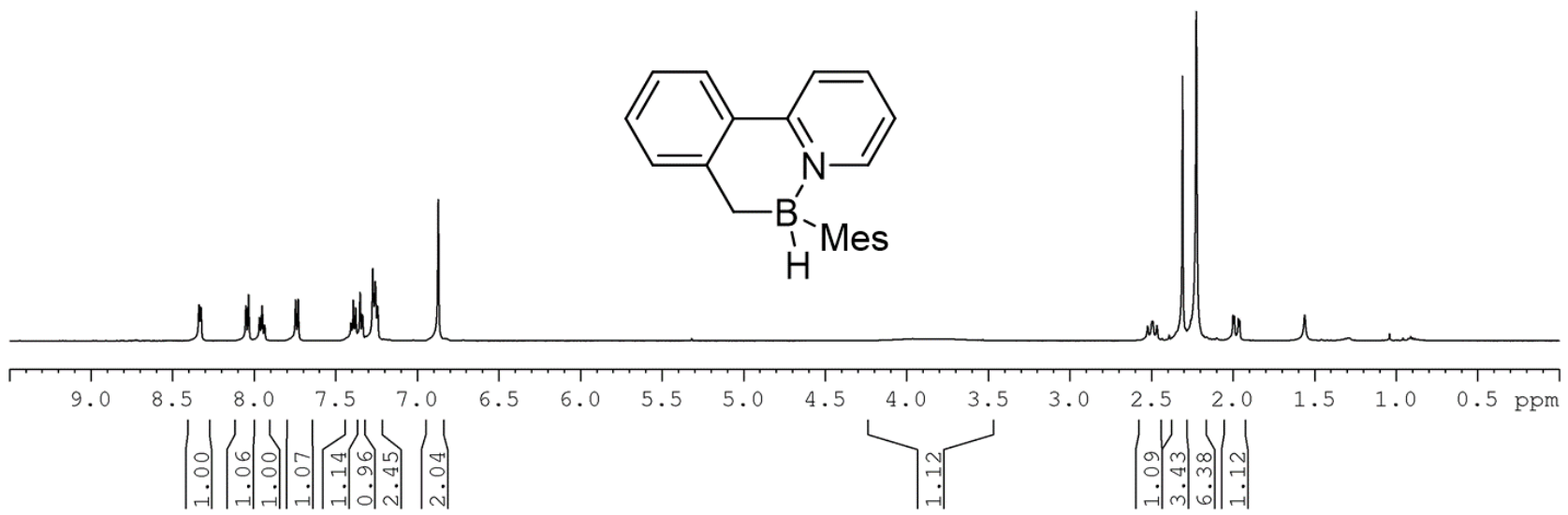

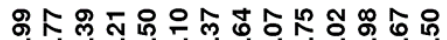

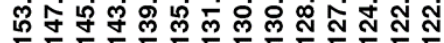

रारा।
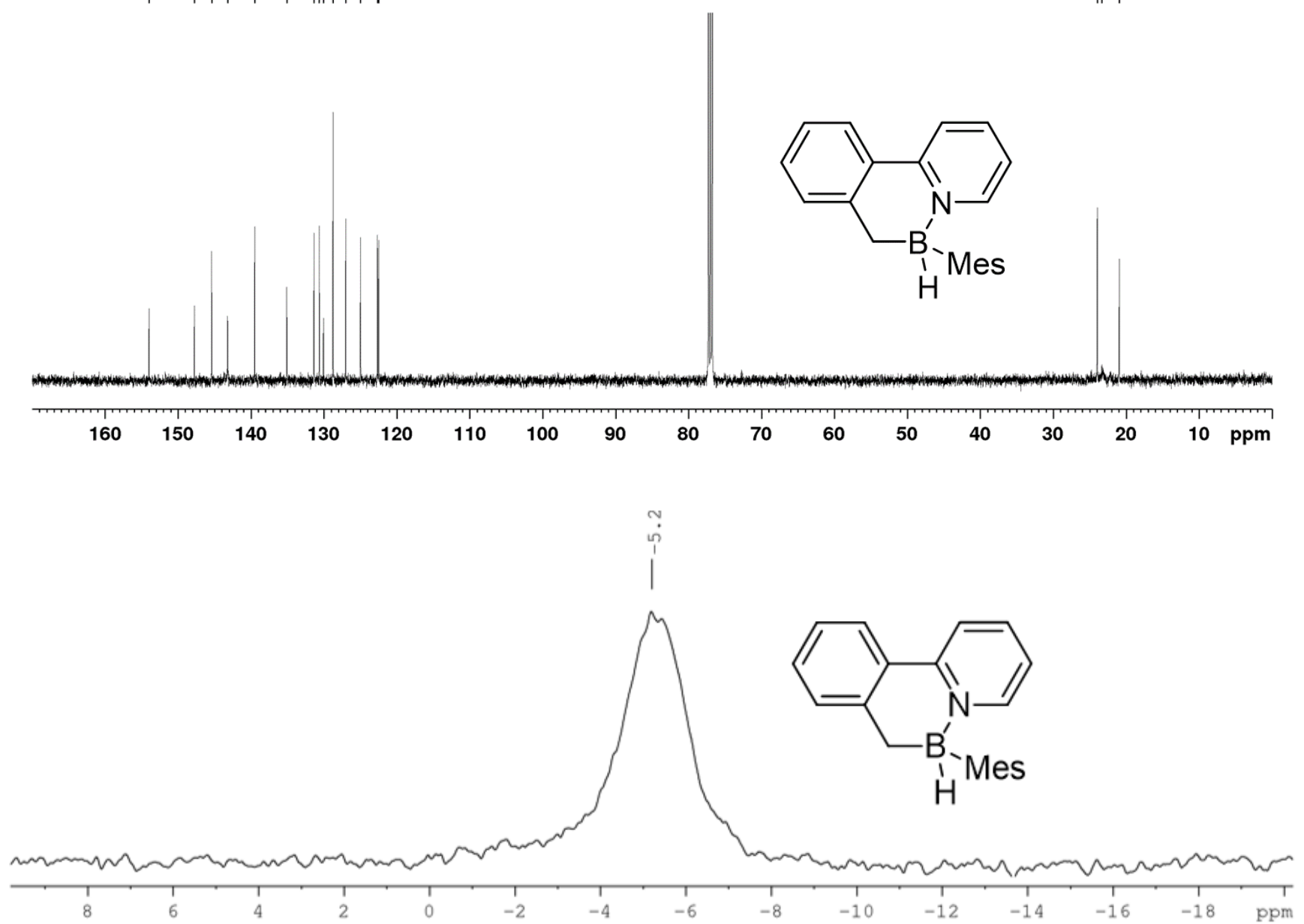
$2.12{ }^{1} \mathrm{H}\left(400 \mathrm{MHz}, \mathrm{C}_{6} \mathrm{D}_{6}\right),{ }^{13} \mathrm{C}\left(126 \mathrm{MHz}, \mathrm{CDCl}_{3}\right)$ and ${ }^{11} \mathrm{~B}\left(160 \mathrm{MHz}, \mathrm{CDCl}_{3}\right)$ NMR of $\mathrm{BN}-$ heterocycle DBN-11
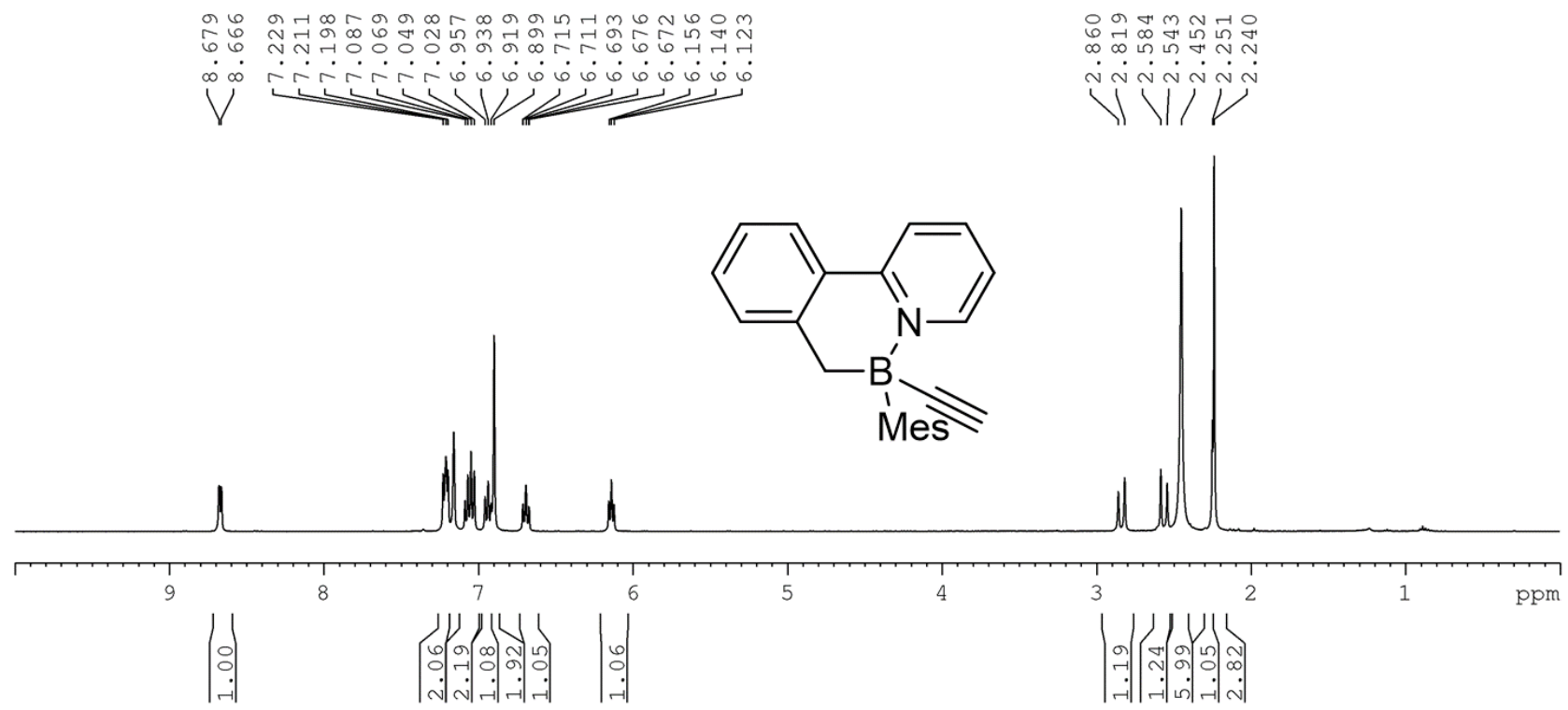

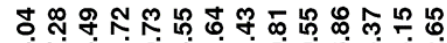

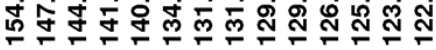

g్
0
0
0
$\mid$

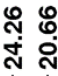
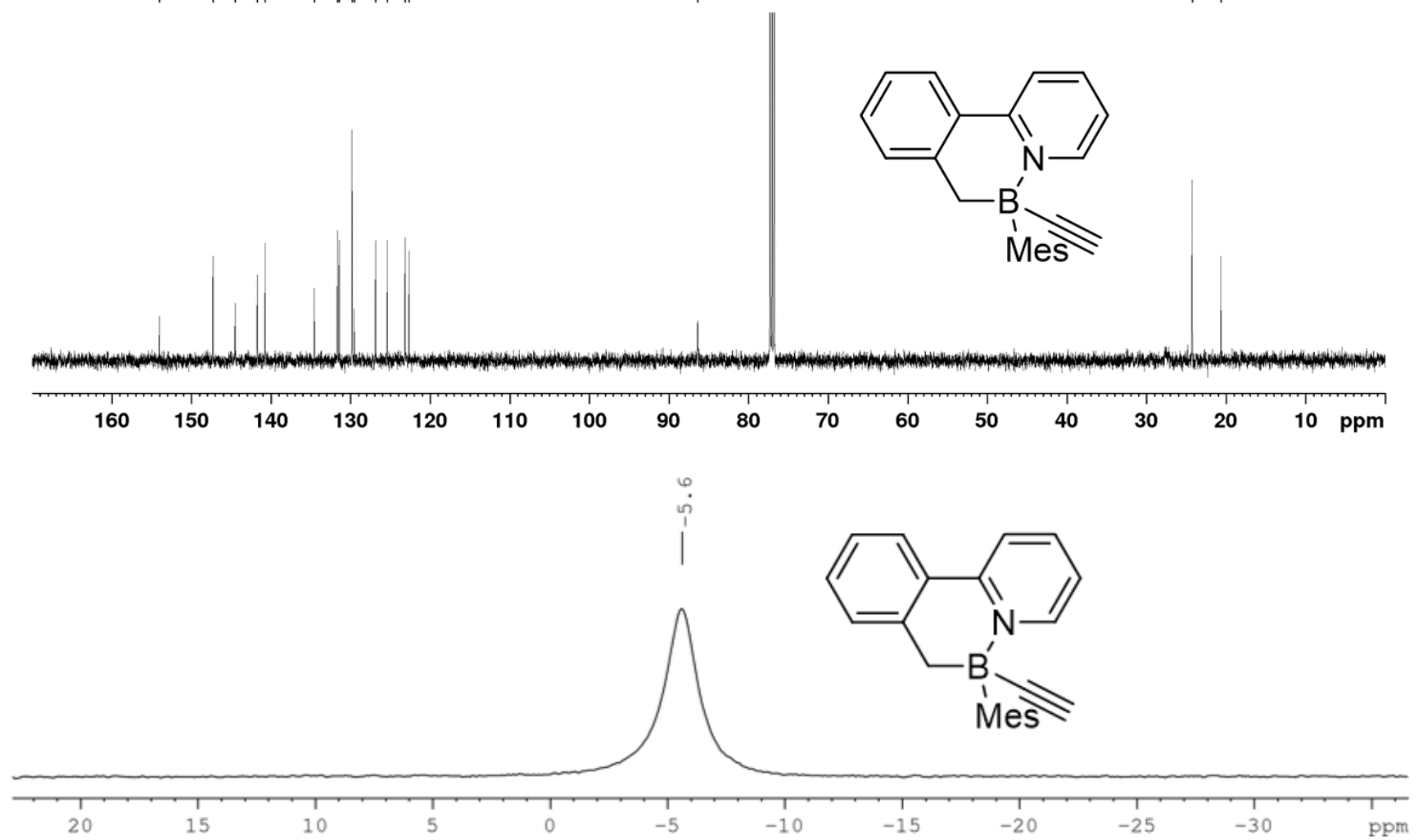
$2.13{ }^{1} \mathrm{H}(400 \mathrm{MHz}){ }^{13} \mathrm{C}(101 \mathrm{MHz})$ and ${ }^{11} \mathrm{~B}(128 \mathrm{MHz}) \mathrm{NMR}$ of BN-heterocycle DBN-11-Ph in $\mathrm{CDCl}_{3}$

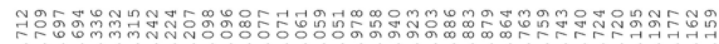
$\underbrace{\infty 00000}$

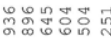

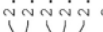
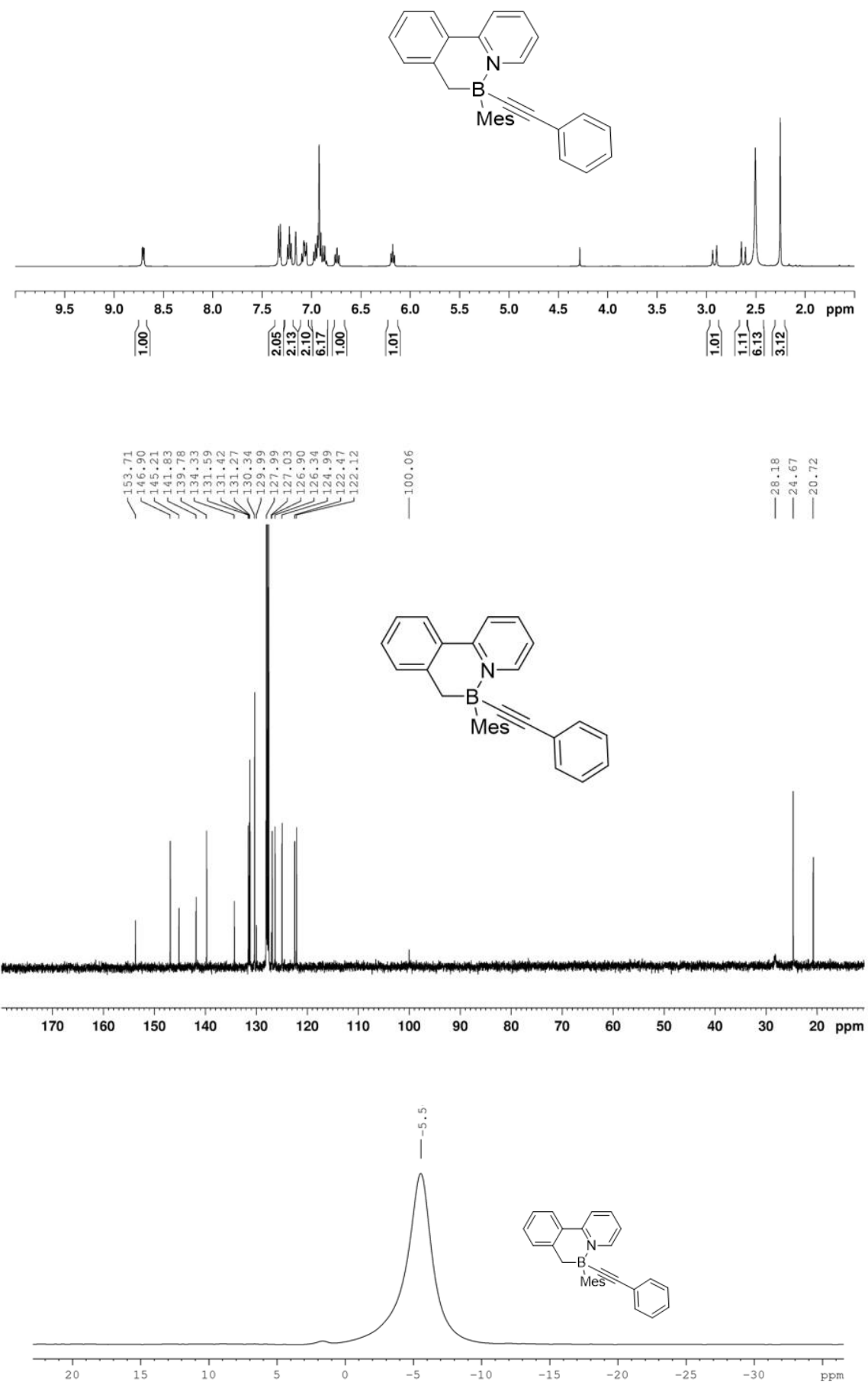


\section{$2.14{ }^{1} \mathrm{H}(400 \mathrm{MHz}),{ }^{13} \mathrm{C}(101 \mathrm{MHz})$ and ${ }^{11} \mathrm{~B}(128 \mathrm{MHz}) \mathrm{NMR}$ of BN-heterocycle DBN-12 in CDCl $\mathrm{C}_{3}$}

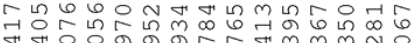

$\infty$

W $/$ W W
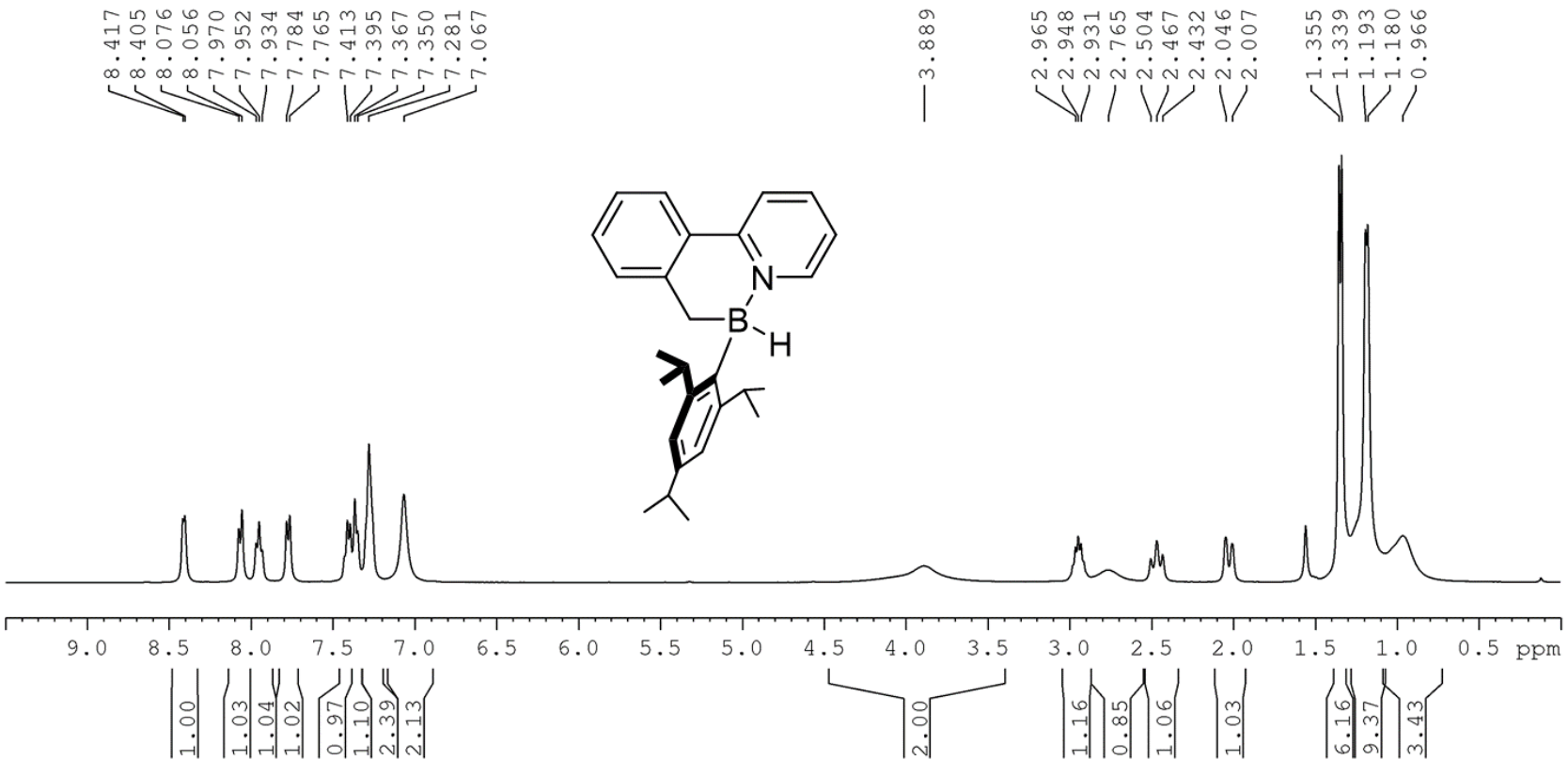

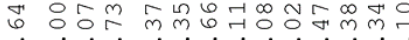

mon

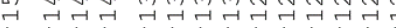

1111111111

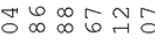

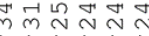

$11 K$
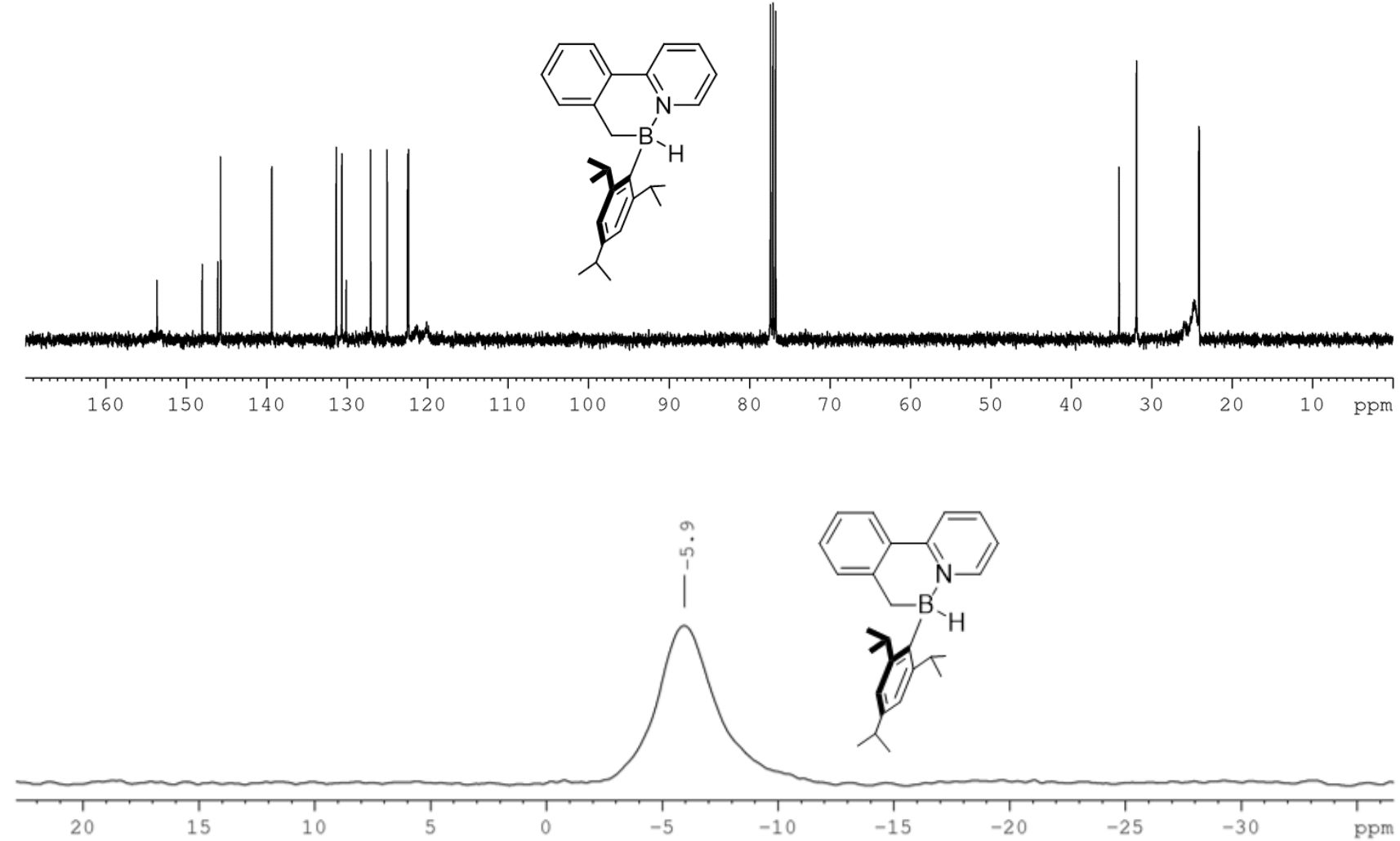


\section{$2.15{ }^{1} \mathrm{H}(400 \mathrm{MHz}),{ }^{13} \mathrm{C}(101 \mathrm{MHz})$ and ${ }^{11} \mathrm{~B}(128 \mathrm{MHz}) \mathrm{NMR}$ of $\mathrm{BN}$-heterocycle DBN-13 in $\mathrm{C}_{6} \mathrm{D}_{6}$}
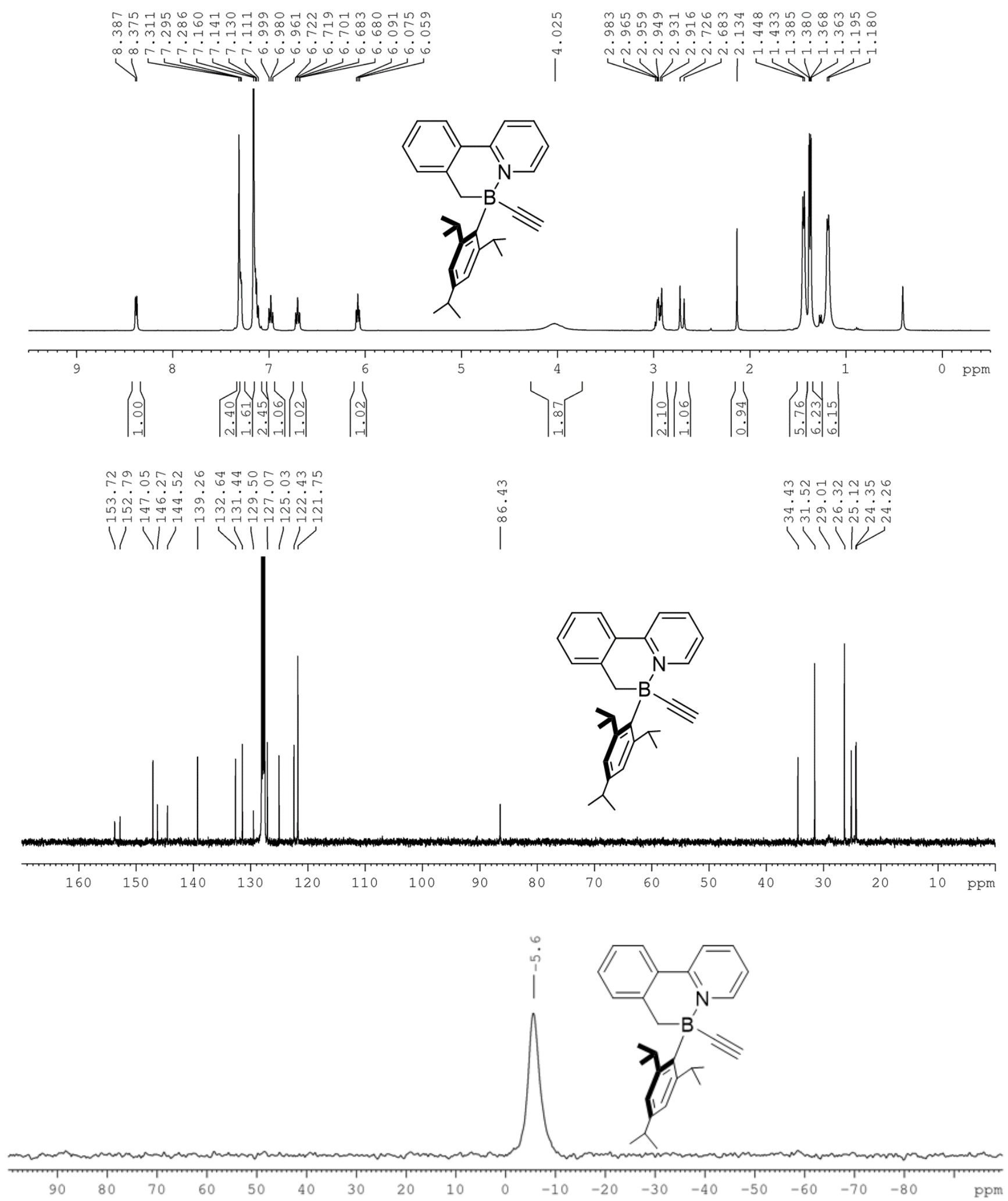


\section{$2.16{ }^{1} \mathrm{H}(400 \mathrm{MHz}),{ }^{13} \mathrm{C}(101 \mathrm{MHz})$ and ${ }^{11} \mathrm{~B}(128 \mathrm{MHz}) \mathrm{NMR}$ of BN-heterocycle DBN-14 in $\mathrm{CDCl}_{3}$}

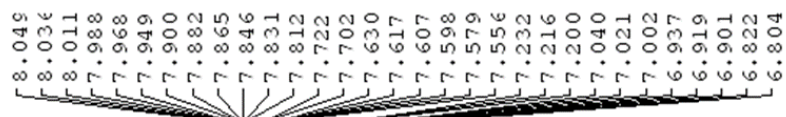

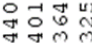

$\dot{m} \dot{m}$

)

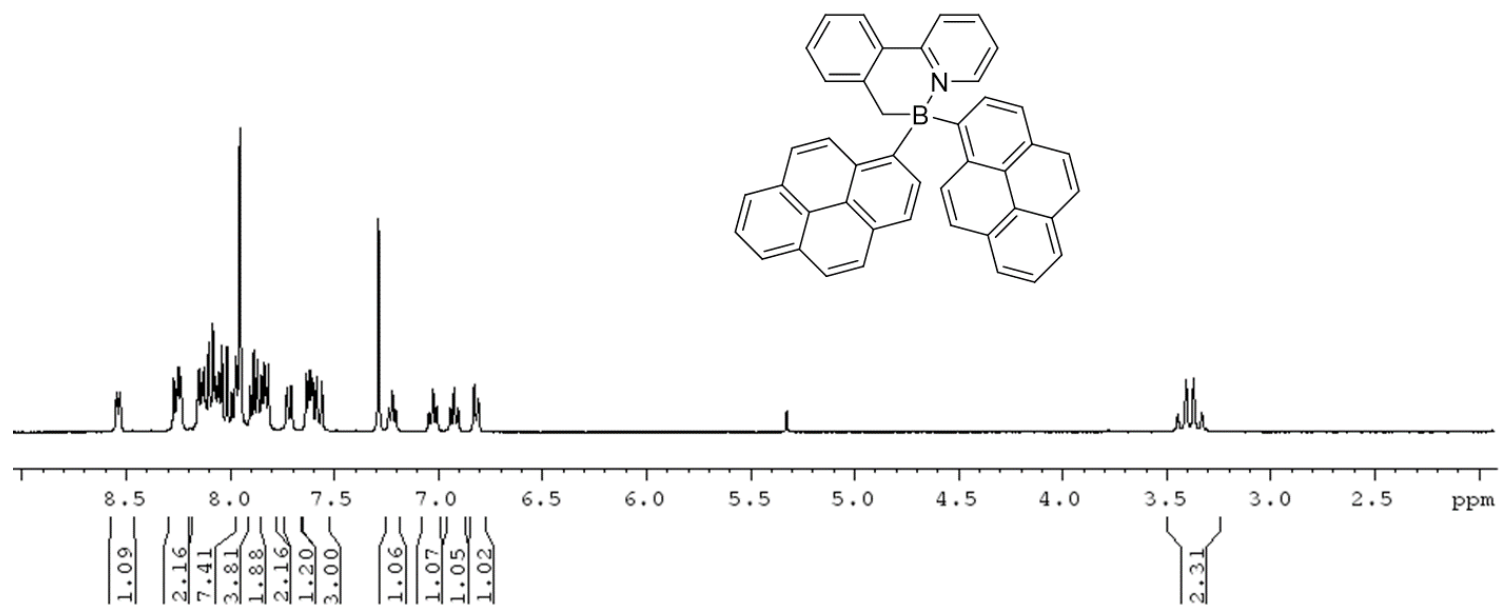

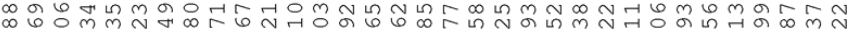

苻
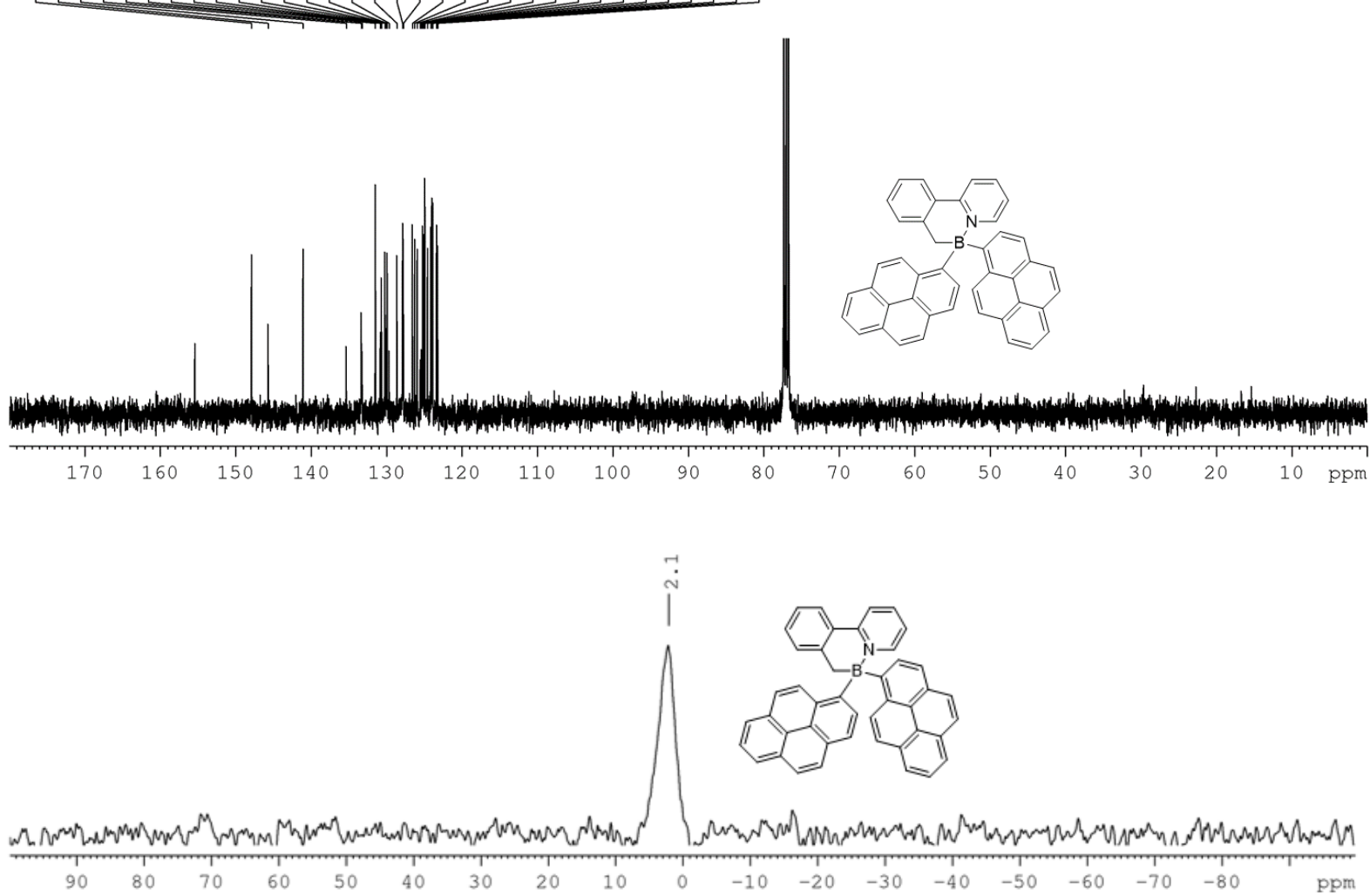
$2.17{ }^{1} \mathrm{H}\left(400 \mathrm{MHz}, \mathrm{CDCl}_{3}\right),{ }^{13} \mathrm{C}\left(101 \mathrm{MHz} \mathrm{CDCl}_{3}\right)$ and ${ }^{11} \mathrm{~B}\left(128 \mathrm{MHz}, \mathrm{C}_{6} \mathrm{D}_{6}\right) \mathrm{NMR}$ of $\mathrm{BN}$-heterocycle DBN-15
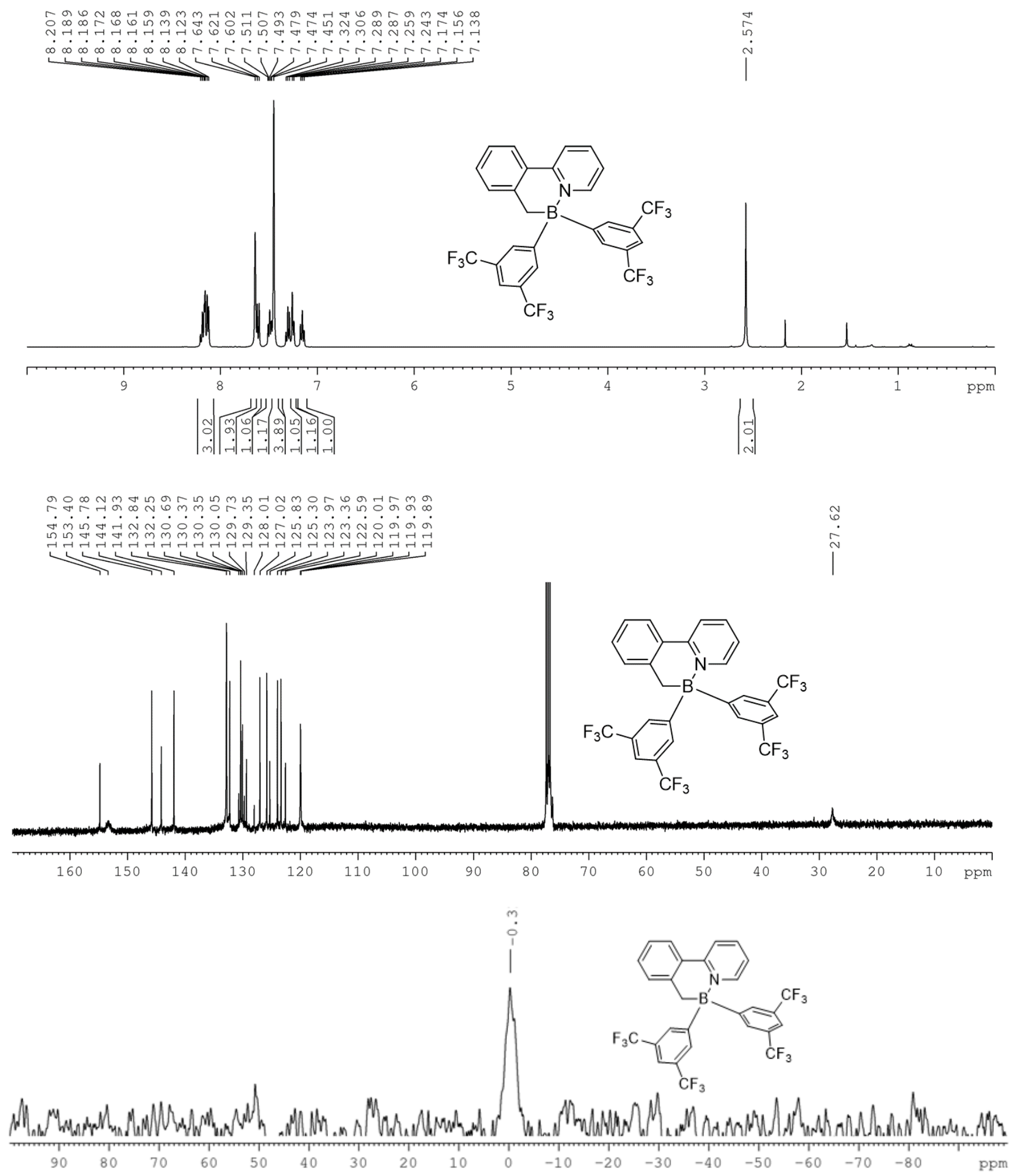
$2.18{ }^{1} \mathrm{H}\left(400 \mathrm{MHz}, \mathrm{CDCl}_{3}\right),{ }^{13} \mathrm{C}\left(101 \mathrm{MHz} \mathrm{CDCl}_{3}\right)$ and ${ }^{11} \mathrm{~B}\left(128 \mathrm{MHz}, \mathrm{C}_{6} \mathrm{D}_{6}\right)$ NMR of $\mathrm{BN}-$ heterocycle DBN-16
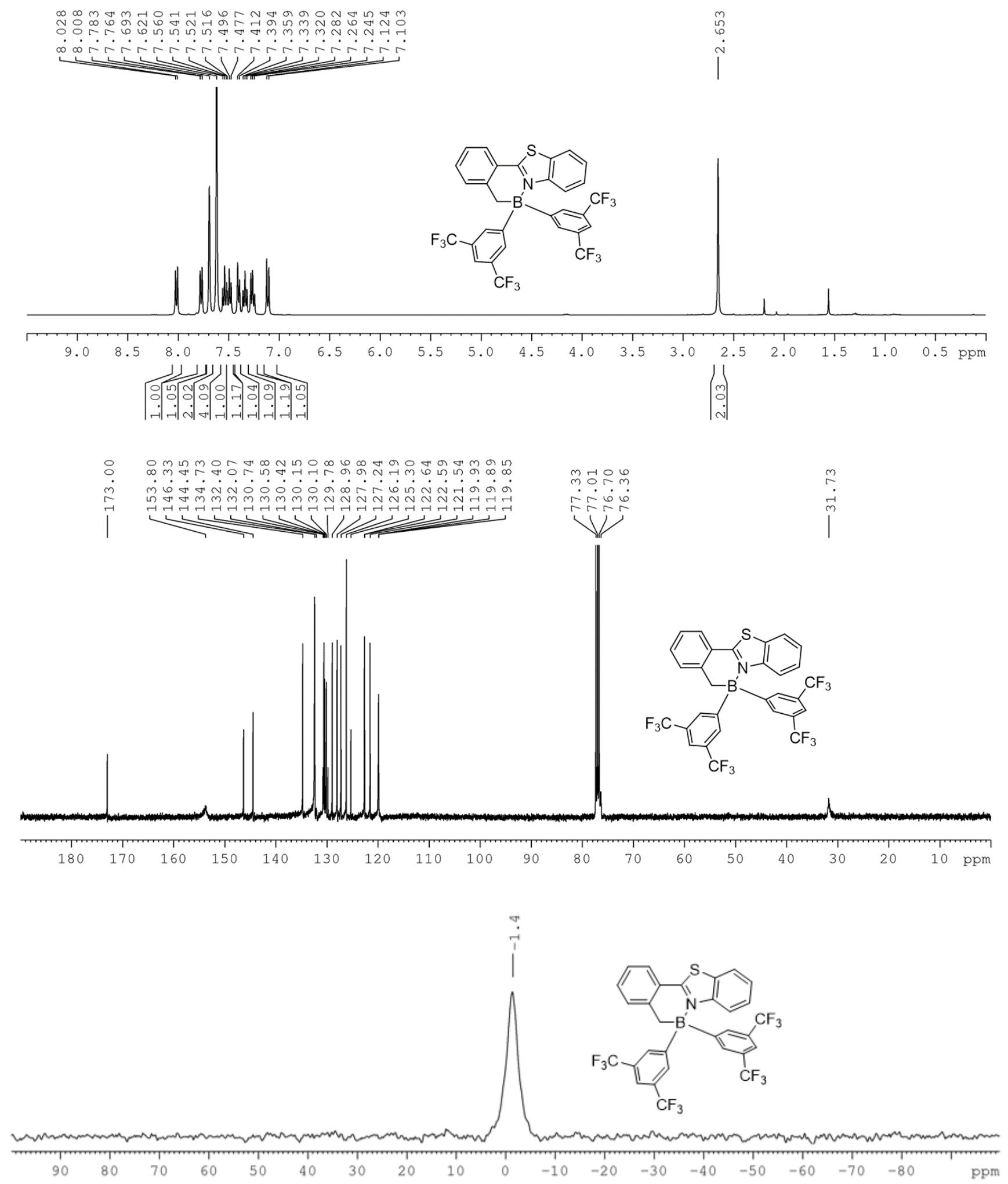
$2.19{ }^{1} \mathrm{H}\left(400 \mathrm{MHz}, \mathrm{CDCl}_{3}\right),{ }^{13} \mathrm{C}\left(101 \mathrm{MHz} \mathrm{CDCl}_{3}\right)$ and ${ }^{11} \mathrm{~B}\left(128 \mathrm{MHz}, \mathrm{C}_{6} \mathrm{D}_{6}\right) \mathrm{NMR}$ of $\mathrm{BN}-$ heterocycle DBN-17

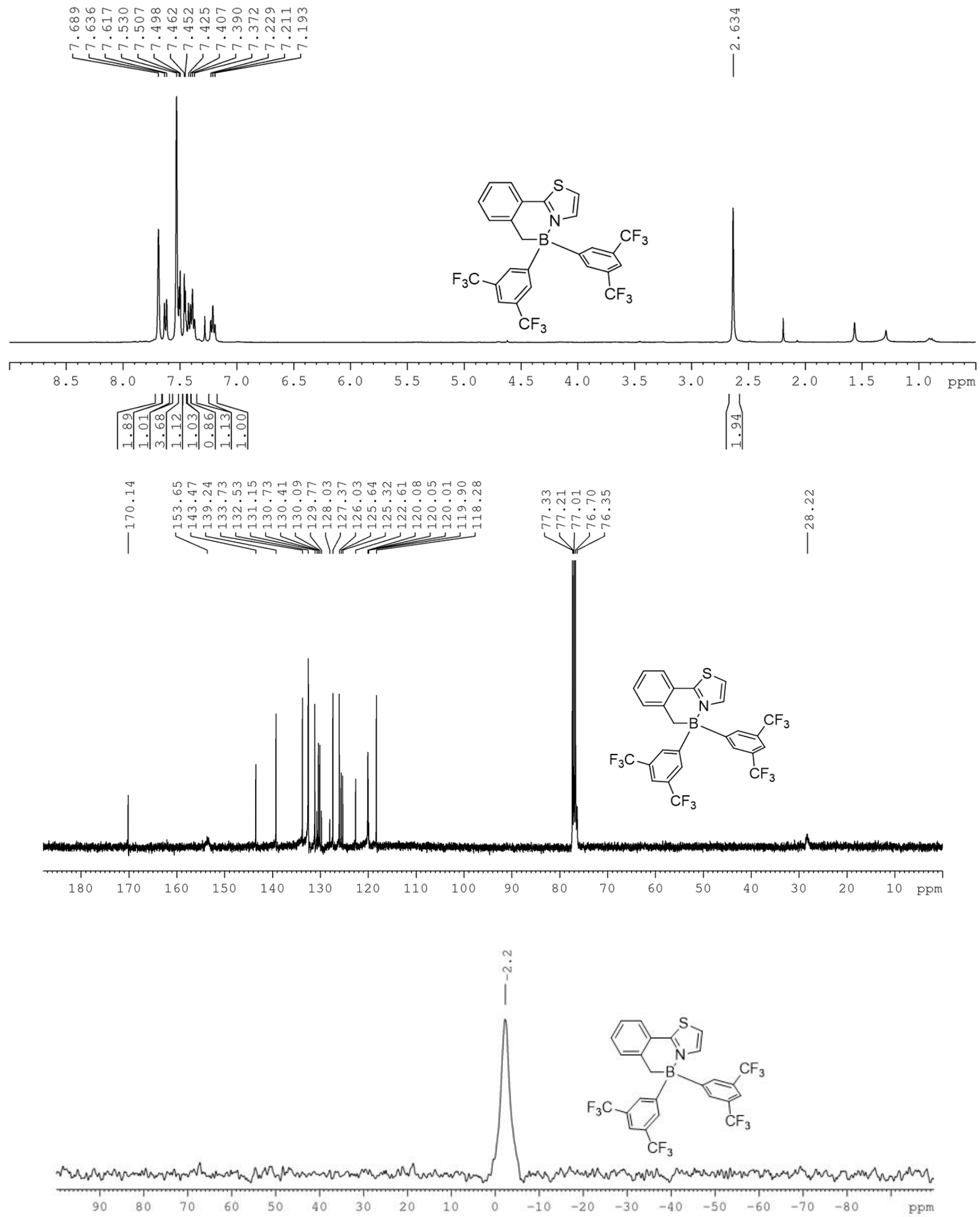




\section{$2.20{ }^{1} \mathrm{H}\left(400 \mathrm{MHz}, \mathrm{CDCl}_{3}\right),{ }^{13} \mathrm{C}\left(101 \mathrm{MHz}, \mathrm{CDCl}_{3}\right)$ and ${ }^{11} \mathrm{~B}\left(128 \mathrm{MHz}, \mathrm{C}_{6} \mathrm{D}_{6}\right) \mathrm{NMR}$ of $\mathrm{BN}-$ heterocycle DBN-18}
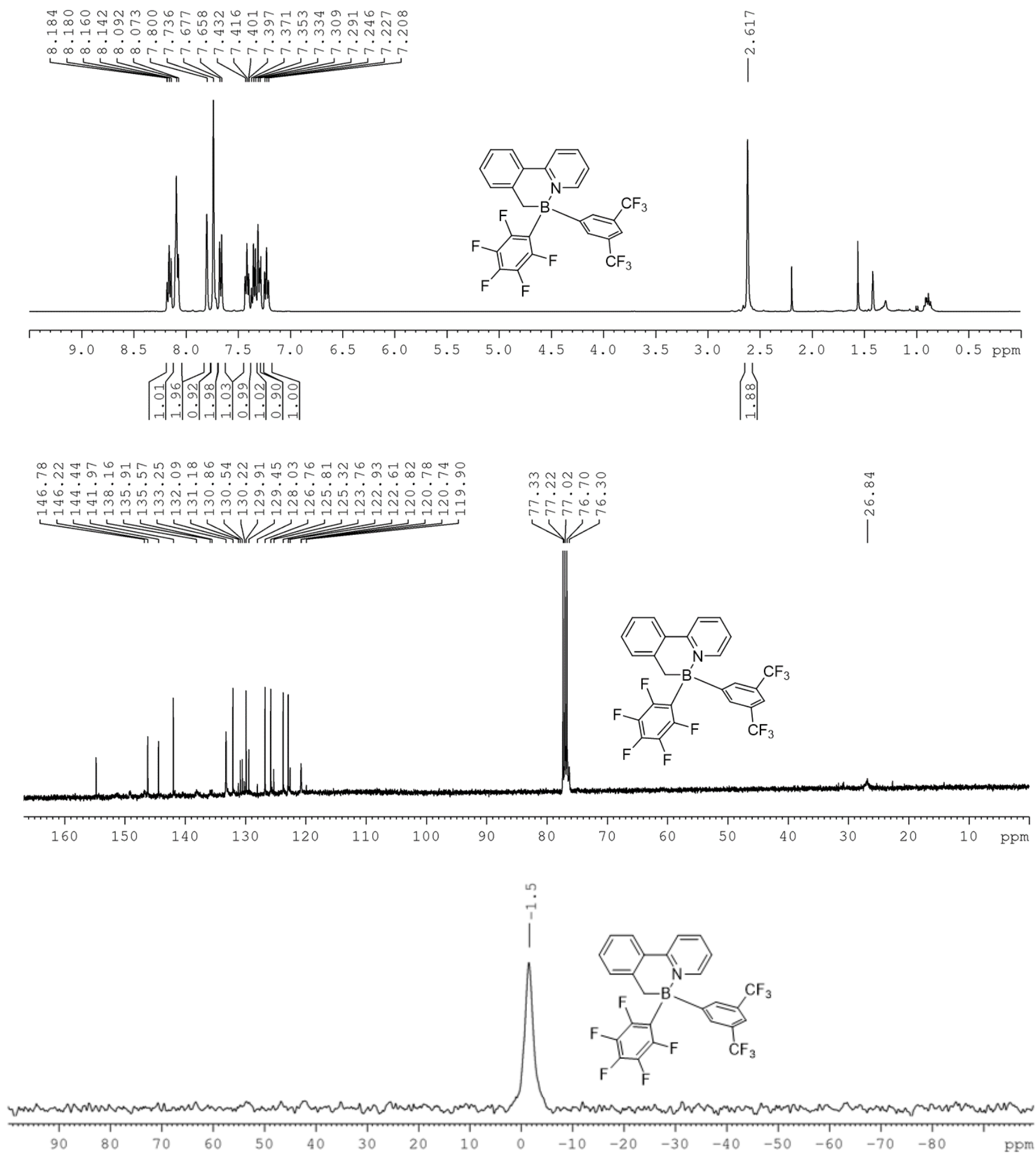
$2.21{ }^{1} \mathrm{H}\left(499 \mathrm{MHz}, \mathrm{C}_{6} \mathrm{D}_{6}\right),{ }^{13} \mathrm{C}\left(126 \mathrm{MHz}, \mathrm{CDCl}_{3}\right)$ and ${ }^{11} \mathrm{~B}\left(160 \mathrm{MHz}, \mathrm{C}_{6} \mathrm{D}_{6}\right)$ NMR of BNheterocycle DBN-19

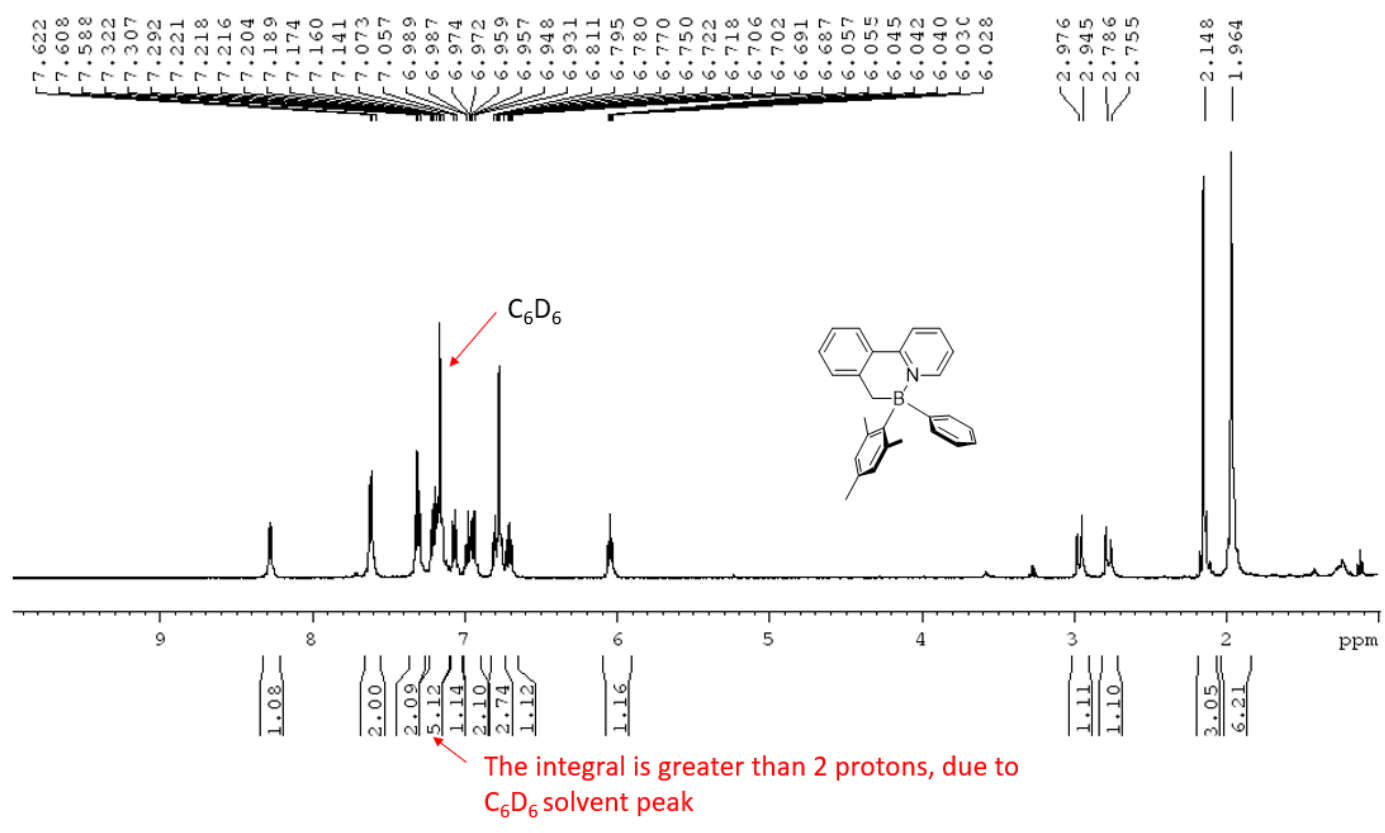

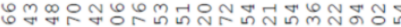

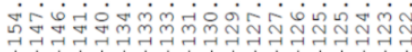

(N)

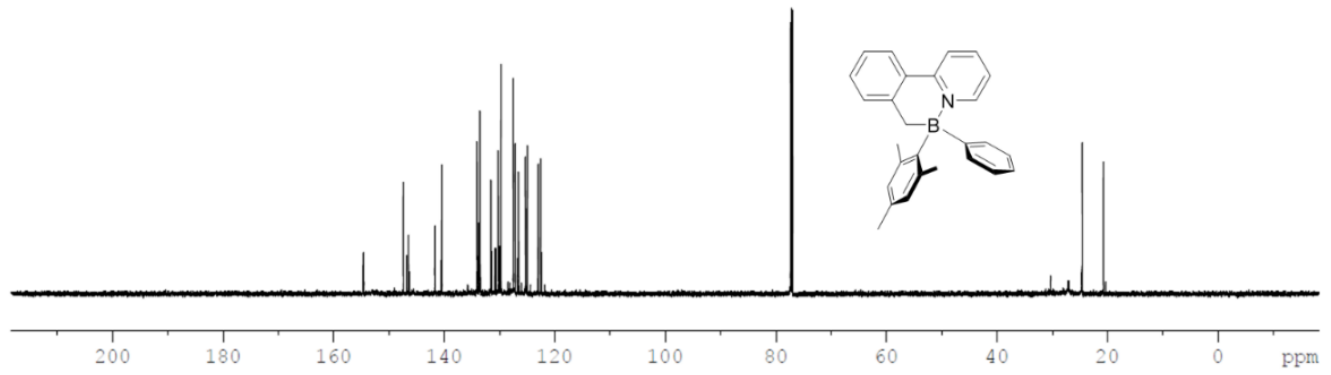

$\stackrel{4}{i}$

|

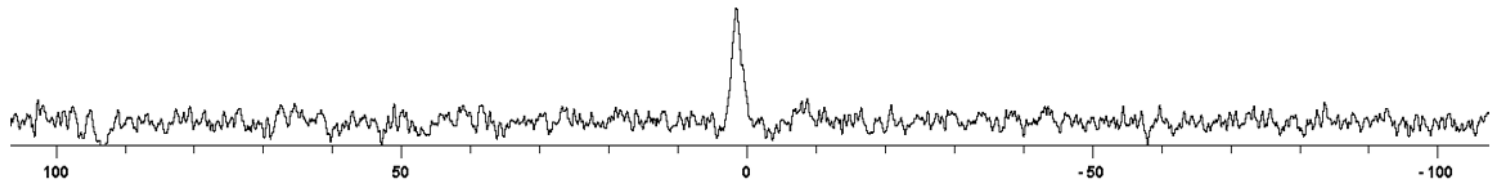




\section{$2.22{ }^{1} \mathrm{H}\left(400 \mathrm{MHz}, \mathrm{C}_{6} \mathrm{D}_{6}\right)$, NMR of DBN-1a}
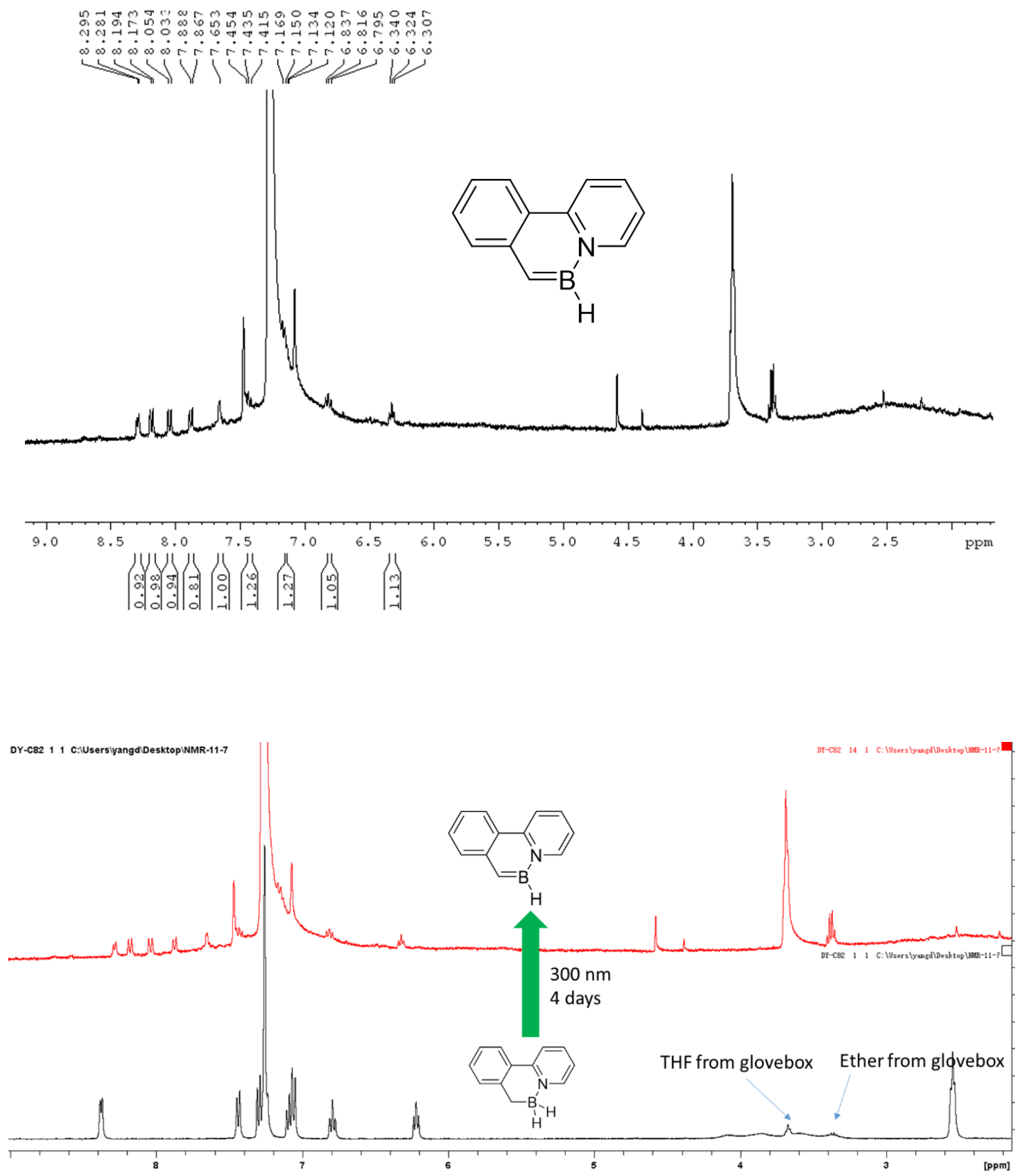


\section{$2.23{ }^{1} \mathrm{H}\left(400 \mathrm{MHz}, \mathrm{C}_{6} \mathrm{D}_{6}\right)$, NMR of DBN-7a}
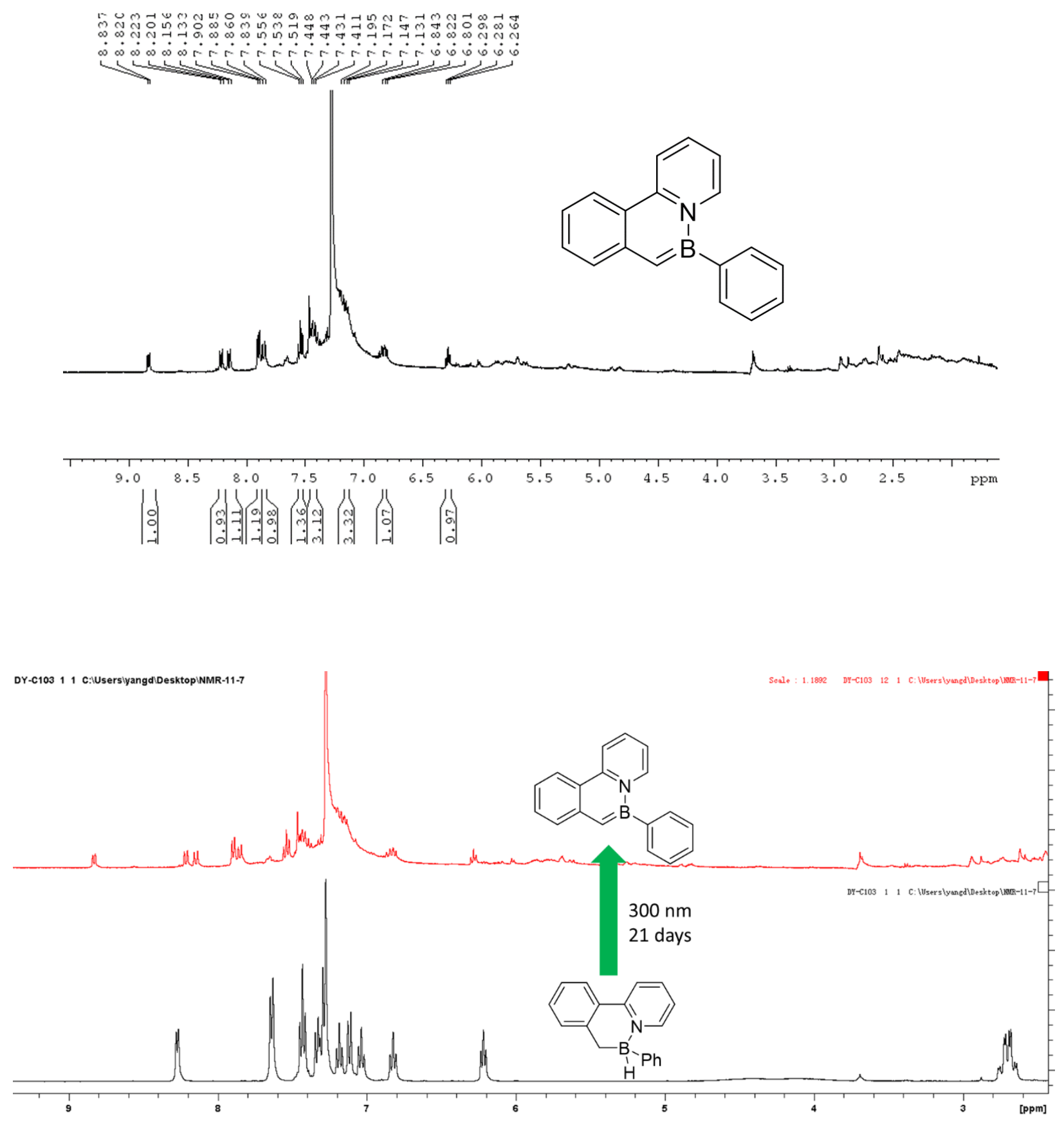


\section{X-ray Crystallographic Data}

Table S1. Crystal data and structure refinement for DBN-3.

Identification code

Empirical formula

Formula weight

Temperature

Wavelength

Crystal system

Space group

Unit cell dimensions

Volume

$\mathrm{Z}$

Density (calculated)

Absorption coefficient

$\mathrm{F}(000)$

Crystal size

Theta range for data collection

Index ranges

Reflections collected

Independent reflections

Completeness to theta $=25.242^{\circ}$

Refinement method

Data / restraints / parameters

Goodness-of-fit on $\mathrm{F}^{2}$

Final $\mathrm{R}$ indices [I>2sigma(I)]

$\mathrm{R}$ indices (all data)

Extinction coefficient

Largest diff. peak and hole
DENGTAO1

C12 H10 B F2 N

217.02

180(2) K

$0.71073 \AA$

Monoclinic

$\mathrm{C} 2 / \mathrm{c}$

$\mathrm{a}=13.7344(7) \AA \quad \alpha=90^{\circ}$.

$\mathrm{b}=7.5557(4) \AA$

$\beta=96.787(2)^{\circ}$.

$c=19.7204(11) \AA$

$\gamma=90^{\circ}$.

2032.10(19) $\AA^{3}$

8

$1.419 \mathrm{Mg} / \mathrm{m}^{3}$

$0.108 \mathrm{~mm}^{-1}$

896

$0.200 \times 0.100 \times 0.100 \mathrm{~mm}^{3}$

2.987 to $27.154^{\circ}$.

$-17<=\mathrm{h}<=16,-8<=\mathrm{k}<=9,-19<=\mathrm{l}<=25$

5740

$2241[\mathrm{R}($ int $)=0.0606]$

$99.9 \%$

Full-matrix least-squares on $\mathrm{F}^{2}$

2241 / 0 / 145

0.907

$\mathrm{R} 1=0.0425, \mathrm{wR} 2=0.0898$

$\mathrm{R} 1=0.0680, \mathrm{wR} 2=0.0994$

$\mathrm{n} / \mathrm{a}$

0.198 and -0.261 e. $\AA^{-3}$ 
Table S2. Crystal data and structure refinement for DBN-10.

\begin{tabular}{|c|c|c|}
\hline Identification code & \multicolumn{2}{|l|}{ DY33 } \\
\hline Empirical formula & \multicolumn{2}{|l|}{$\mathrm{C} 21 \mathrm{H} 22 \mathrm{~B} N$} \\
\hline Formula weight & \multicolumn{2}{|l|}{299.20} \\
\hline Temperature & \multicolumn{2}{|l|}{$296(2) \mathrm{K}$} \\
\hline Wavelength & \multicolumn{2}{|l|}{$0.71073 \AA$} \\
\hline Crystal system & \multicolumn{2}{|l|}{ Orthorhombic } \\
\hline Space group & \multicolumn{2}{|l|}{$\mathrm{P} 2{ }_{1} 2_{1} 2_{1}$} \\
\hline \multirow[t]{3}{*}{ Unit cell dimensions } & $\mathrm{a}=8.0361(2) \AA$ & $\mathrm{a}=90^{\circ}$ \\
\hline & $\mathrm{b}=12.9083(4) \AA$ & $\mathrm{b}=90^{\circ}$ \\
\hline & $\mathrm{c}=15.8908(5) \AA$ & $\mathrm{g}=90^{\circ}$. \\
\hline Volume & \multicolumn{2}{|l|}{$1648.39(8) \AA^{3}$} \\
\hline $\mathrm{Z}$ & \multicolumn{2}{|l|}{4} \\
\hline Density (calculated) & \multicolumn{2}{|l|}{$1.206 \mathrm{Mg} / \mathrm{m}^{3}$} \\
\hline Absorption coefficient & \multicolumn{2}{|l|}{$0.068 \mathrm{~mm}^{-1}$} \\
\hline $\mathrm{F}(000)$ & \multicolumn{2}{|l|}{640} \\
\hline Crystal size & \multicolumn{2}{|c|}{$0.140 \times 0.060 \times 0.060 \mathrm{~mm}^{3}$} \\
\hline Theta range for data collection & \multicolumn{2}{|l|}{2.033 to $27.111^{\circ}$. } \\
\hline Index ranges & \multicolumn{2}{|c|}{$-10<=\mathrm{h}<=10,-16<=\mathrm{k}<=15,-20<=\mathrm{l}<=17$} \\
\hline Reflections collected & \multicolumn{2}{|l|}{14855} \\
\hline Independent reflections & \multicolumn{2}{|c|}{$3624[\mathrm{R}(\mathrm{int})=0.0533]$} \\
\hline Completeness to theta $=25.242^{\circ}$ & \multicolumn{2}{|l|}{$100.0 \%$} \\
\hline Absorption correction & \multicolumn{2}{|c|}{ Semi-empirical from equivalents } \\
\hline Max. and min. transmission & \multicolumn{2}{|l|}{0.7455 and 0.6910} \\
\hline Refinement method & \multicolumn{2}{|c|}{ Full-matrix least-squares on $\mathrm{F}^{2}$} \\
\hline Data / restraints / parameters & \multicolumn{2}{|l|}{3624 / 0 / 212} \\
\hline Goodness-of-fit on $\mathrm{F}^{2}$ & \multicolumn{2}{|l|}{1.045} \\
\hline Final $\mathrm{R}$ indices $[\mathrm{I}>2 \operatorname{sigma}(\mathrm{I})]$ & \multicolumn{2}{|c|}{$\mathrm{R} 1=0.0451, \mathrm{wR} 2=0.0984$} \\
\hline $\mathrm{R}$ indices (all data) & \multicolumn{2}{|c|}{$\mathrm{R} 1=0.0640, \mathrm{wR} 2=0.1093$} \\
\hline Absolute structure parameter & \multicolumn{2}{|l|}{$2(5)$} \\
\hline Extinction coefficient & \multicolumn{2}{|l|}{$\mathrm{n} / \mathrm{a}$} \\
\hline Largest diff. peak and hole & \multicolumn{2}{|c|}{0.179 and $-0.184 \mathrm{e} . \AA^{-3}$} \\
\hline
\end{tabular}

Largest diff. peak and hole 
Table S3. Crystal data and structure refinement for jb3_sq.

Identification code

Empirical formula

Formula weight

Temperature

Wavelength

Crystal system

Space group

Unit cell dimensions

Volume

Z

Density (calculated)

Absorption coefficient

$\mathrm{F}(000)$

Crystal size

Theta range for data collection

Index ranges

Reflections collected

Independent reflections

Completeness to theta $=25.242^{\circ}$

Absorption correction

Max. and min. transmission

Refinement method

Data / restraints / parameters

Goodness-of-fit on $\mathrm{F}^{2}$

Final R indices [I $>2 \operatorname{sigma}(\mathrm{I})]$

$\mathrm{R}$ indices (all data)

Extinction coefficient

Largest diff. peak and hole jb3_sq

C88 H56 B2 N2

1162.96

296(2) K

$0.71073 \AA$

Triclinic

P-1

$\mathrm{a}=11.8767(2) \AA$

$\mathrm{a}=95.2000(10)^{\circ}$.

$\mathrm{b}=16.8709(3) \AA$

$\mathrm{b}=103.7540(10)^{\circ}$.

$c=18.0360(3) \AA$

$\mathrm{g}=108.7830(10)^{\circ}$.

3267.93(10) $\AA^{3}$

2

$1.182 \mathrm{Mg} / \mathrm{m}^{3}$

$0.067 \mathrm{~mm}^{-1}$

1216

$0.100 \times 0.100 \times 0.050 \mathrm{~mm}^{3}$

1.296 to $27.147^{\circ}$.

$-15<=\mathrm{h}<=15,-21<=\mathrm{k}<=21,-23<=1<=23$

54775

$14388[\mathrm{R}(\mathrm{int})=0.0581]$

$99.9 \%$

Semi-empirical from equivalents

0.7455 and 0.6936

Full-matrix least-squares on $\mathrm{F}^{2}$

14388 / 0 / 829

1.006

$\mathrm{R} 1=0.0515, \mathrm{wR} 2=0.1068$

$\mathrm{R} 1=0.1246, \mathrm{wR} 2=0.1310$

$\mathrm{n} / \mathrm{a}$

0.151 and -0.173 e. $\AA^{-3}$ 